\title{
Impact of Different Shelter Designs on Haematological Profile in Dairy Cattle of Tamil Nadu, India
}

\author{
A. Paramasivam ${ }^{1}$, P.S.L. Sesh ${ }^{2}$, T. Sivakumar ${ }^{1}$, P. Tensingh Gnanaraj ${ }^{1}$ and P.T. Suraj ${ }^{1}$ \\ ${ }^{1}$ Department of Livestock Production and Management, ${ }^{2}$ Department of Veterinary \\ Biochemistry, Madras Veterinary College, Chennai, Tamil Nadu Veterinary and Animal \\ Sciences University, Tamil Nadu - 600 007, India \\ *Corresponding author
}

\section{A B S T R A C T}

A study was carried out to assess the impact of shelter designs on the production performance of dairy cattle in the three selected agro - climatic zones of Tamil Nadu, viz. Southern, Cauvery delta and High rainfall zones. The effects of various shelter designs on

\section{Keywords}

Shelter designs,

Haematological parameters, Dairy cattle

Article Info

Accepted:

10 January 2019

Available Online:

10 February 2019 the haematological parameters were analyzed. The whole blood samples were collected throughout the year i.e. in all the four seasons, viz., winter, summer, south-west monsoon and north-east monsoon. For each season, a total of thirty samples (in duplicate) were collected from five housing types, each comprising of six individual cattle from the three agro-climatic regions for measuring the haematological profile. The blood parameters, namely WBC count, RBC count, Hb, HCT, MCV, MCH, MCHC, RDW, PLT, MPV, PDW and PCT were estimated using automated haematology analyzer in all the above samples. The results of $\mathrm{WBC}$ count, $\mathrm{MCH}, \mathrm{MCHC}$ and PCT values showed significant difference between all the four seasons in all the three agro-climatic zones, while the results of RBC count, $\mathrm{Hb}$, HCT differed significantly between all the four seasons in Cauvery delta and Southern zones and PLT values differed significantly between seasons in the Southern and High rainfall zones. With respect to housing types, besides the seasonal variation mentioned above, the results of MCV differed significantly in Cauvery delta and High rainfall zones, while the results of WBC count and MCHC differed significantly only in Cauvery delta zone. The interaction between the influence of Seasons and Housing types on haematological profile showed significant differences for PDW in High rainfall zone and for PCT in Cauvery delta zone. Factor analysis study of this haematological profile with other climatic, biochemical and production parameters in all the three zones revealed that in Southern and Cauvery delta zones open housing is best and in High rainfall zone thatched roof system is the best.

\section{Introduction}

In Tamil Nadu, livestock sector contributes about 2.74 per cent of Gross State Domestic Product and the annual milk production in the state during the year 2015-16 was 7244 thousand tonnes. The state contributes about 5.31 per cent of milk production to the national milk grid and stands $9^{\text {th }}$ in milk production in the country (2015-16, NDDB Report). 
Dairy farming is perceived by the farmers of our country as a part of an integrated agricultural system, where dairy and agriculture complement each other. In Dairying, housing management along with feeding plays a very important role in exploiting the genetic potential of dairy cows. Optimal feeding and housing are the prerequisite factor for milk production. To utilize the feed more efficiently the housing management becomes important which helps to maintain the thermo neutral zone in which the animals are most productive. The environmental conditions of livestock buildings significantly affect animal welfare and productivity.

The cow shelters under Indian conditions are designed to reduce the heat load because heat stress causes more stress to animals compared to winter. Principles for creating an optimum micro environment in and around the sheds are aimed to reduce heat gain and promote heat losses. In order to ensure optimum productivity of the dairy cattle, understanding of the existing housing patterns and the environmental stress in various agro-climatic regions is very essential. The most important environmental interventions done in recent days are those that have been done in housing and other attempts to ameliorate the thermal extremes. An environment in which stress is minimized would likely be favourable for efficient production of products derived from domestic farm animals and for helping ensure the well-being of those species.

Hence a study was carried out to assess the impact of shelter designs on the production performance of dairy cattle in the three selected agro - climatic zones of Tamil Nadu. viz. Southern, Cauvery delta and High rainfall zones. In this communication, the effects of various shelter designs on haematological parameters are discussed.

\section{Materials and Methods}

The research work was carried out in two phases. The first one involved the base line survey to identify the existing dairy cattle housing system along with management practices followed by farmers in the representative districts from Southern (Madurai, Pudukkottai, Dindigul, Virudhunagar and Tirunelveli Districts), Cauvery delta (Trichy and Thanjavur Dist.) and High rainfall (Kanyakumari Dist.) zones.

The second one involved the seasonal stress assessment of the cattle in the selected farms from each zone, throughout the year i.e. all the four seasons, viz., winter, summer, southwest monsoon and north-east monsoon.

\section{Collection of samples}

In each of the four seasons, a total of thirty samples (in duplicate) were collected from five housing types (open housing, thatch roofed, asbestos roofed, tile roofed and metal sheet housing), with each housing type comprising of six individual cattle $(6 \times 5=30)$ for measuring the haematological profile from the three agro-climatic regions. Thus, a total of 90 samples were collected during each season with a grand total of 360 samples (i.e. $30 \times 3 \times 4=360$ ) during the whole experimental period of one year. In each sampling season, the number of animals sampled in each group was always greater than five as recommended by Whitaker (2000).

Whole blood was collected between 12.00 noon to $2.00 \mathrm{pm}$ from each cow through jugular vein puncture into blood collecting vials coated with EDTA for haematological studies as described by Grunwaldt, et al., (2005) and was stored at $4{ }^{\circ} \mathrm{C}$ till analysis. 


\section{Haematological Studies}

The following blood parameters were studied by standard methods described by Coles (1986), Sastry et al., (1989) and Perumal et al., (2010) using automated haematology analyzer (Mindray, BC- 2800 Vet, Lab Resource, Inc., USA).

i. White Blood Cell (WBC) Count / Total Leukocyte Count - WBC Count / TLC

ii. Red Blood Cell (RBC) Count / Total Erythrocyte Count - RBC Count / TEC

iii. Haemoglobin Concentration - $\mathrm{Hb}$

iv. Haematocrit Value - HCT / PCV

v. Mean Corpuscular Volume - MCV

vi. Mean Corpuscular Haemoglobin - MCH

vii. Mean Corpuscular Haemoglobin Concentration - MCHC

viii. Red Blood Cell Distribution Width RDW

ix. Platelet Count - PLT Count

x. Mean Platelet Volume - MPV

xi. Platelet Distribution Width - PDW

xii. Platelet Crit Value - PCT

\section{Statistical analysis}

The experimental data were statistically analyzed by IBM SPSS Statistics Version 20.0 by using statistical tools two way analysis using General Linear Model Univariate Procedure. The significance was tested by using Tukeys HSD and T Test.

\section{Results and Discussion}

\section{WBC count}

The mean \pm S.E of WBC value of animals under various housing types in all the agroclimatic zones under the study are presented in the Table 1. WBC count was found to differ significantly $(\mathrm{P}<0.05)$ between different seasons in the Southern and Cauvery delta zones. High rainfall zone showed highly significant difference $(\mathrm{P}<0.01)$ between seasons for WBC level. Thatched shed in high rainfall zone showed higher WBC $(20.5 \pm 3.9$ $\left.10^{3} / \mu 1\right)$ count during North East monsoon. This may be due to bacterial diseases arising out of heavy rainfall received in High rainfall zone during two monsoons. Whereas, Tiled shed in Southern zone showed lower WBC count $\left(6.1 \pm 0.810^{3} / \mu 1\right)$ during summer which may be due to summer stress. Total mean value of WBC for all the zones ranged between $9.3 \pm 0.5$ to $15.3 \pm 1.610^{3} / \mu 1$ and it was found to differ with the findings of Sinha et al., (2009) who recorded the mean value of WBC in Central Uttarpradesh falls between $7.55 \pm 0.09$ to $8.59 \pm 0.2110^{3} / \mu 1$.

\section{RBC count}

The mean \pm S.E of RBC count of animals under various housing types in all the agroclimatic zones under the study are presented in the Table 1. It can be seen that difference in RBC count was found to be highly significant between seasons in the Southern zone and in Cauvery delta zone $(\mathrm{P}<0.01)$, whereas the difference was not found to be significant in high rainfall zone. Thatched shed in Southern zone recorded higher count of RBC (6.72 \pm $1.2410^{6} / \mu 1$ ) during North East monsoon. Whereas, tiled shed in Cauvery delta zone showed lower RBC count $\left(3.67 \pm 0.1310^{6} / \mu \mathrm{l}\right)$ during summer. Decreased RBC count below the normal level may be possible due to blood loss, haemolysis or decreased production due to nutritional deficiency especially mineral deficiency. This finding (mineral deficiency) concurred with the views reported by Sharma et al., (2009).

\section{Hb concentration}

The mean \pm S.E of $\mathrm{Hb}$ concentration of animals under various housing types in Southern, Cauvery delta and High rainfall zone are presented in the Table 2 . It can be understood that $\mathrm{Hb}$ was found to differ significantly $(\mathrm{P}<0.01)$ between different seasons in the Southern and Cauvery delta 
zones. In all the three zones, neither the season nor the type of housing had any effect on the level of $\mathrm{Hb}$ in milch cows. During North East monsoon, thatched shed in Southern zone showed higher value of $\mathrm{Hb}$ $(9.9 \pm 0.7 \mathrm{~g} / \mathrm{dl})$. In Cauvery delta zone, tiled shed showed lower $\mathrm{Hb}$ content $(5.2 \pm 0.21$ $\mathrm{g} / \mathrm{dl}$ ) during summer which may be due to heat stress. This is in close agreement with the findings of Weldy et al., (1964). In contrast to the present findings, Srikanthakumar and Johnson (2004) reported that heat stress in Australian conditions increases $\mathrm{Hb}$ content in lactating Holstein, Jersey and Australian milking zebu.

\section{HCT value}

The mean \pm S.E of HCT of animals under various housing types in Southern, Cauvery delta and High rainfall zone are presented in the Table 2. It can be seen that HCT was found to differ significantly higher between different seasons in the Southern and Cauvery delta zone $(\mathrm{P}<0.01)$. During North East monsoon season, open shed in Southern zone recorded higher HCT content $(28 \pm 1.0 \%)$, whereas, tiled shed in Cauvery delta zone recorded lower HCT $(15.5 \pm 0.1 \%)$ content during summer. Low level of HCT during summer may be due to breed difference variation (Jersey and Holstein Friesian cows). Similar finding was also reported by Banerjee and Ashutosh (2010).

\section{MCV}

The mean \pm S.E of MCV of animals under various housing types in Southern, Cauvery delta and High rainfall zone are presented in the Table 3. In the present study, it was evident that during South West monsoon season thatched shed in high rainfall zone recorded higher MCV $(52.4 \pm 2.0 \mathrm{fl})$ whereas, metal shed in Southern zone recorded lower MCV (38.5 $\pm 6.7 \mathrm{fl})$ content during summer. Similar finding was reported by Randhawa et al., (2009).

\section{МCH}

The mean \pm S.E of $\mathrm{MCH}$ of animals under various housing types in Southern, Cauvery delta and High rainfall zone are presented in the Table 3. In the present study, it was evident that the difference in $\mathrm{MCH}$ levels between the season was found to be significant $(\mathrm{P}<0.05)$ in all the three zones. During South West monsoon season, asbestos shed in high rainfall zone recorded higher $\mathrm{MCH}(20.7 \pm 4.1 \mathrm{pg})$, whereas, tiled shed in Cauvery delta zone recorded lower $\mathrm{MCH}$ $(13.6 \pm 0.2 \mathrm{pg})$ content during summer. The present study differed from the findings of Randhawa et. al. (2009) who reported that rainy season had significantly lower $\mathrm{MCH}$ content.

\section{MCHC}

The mean \pm S.E of MCHC of animals under various housing types in Southern, Cauvery delta and High rainfall zone are presented in the Table 4. There was a significant difference $(\mathrm{P}<0.01)$ between the seasons in all the zones. Among three zones, Cauvery delta zone alone showed significant difference $(\mathrm{P}<0.01)$ between the housing types. During South West monsoon season, asbestos shed in Southern zone recorded higher $\mathrm{MCHC}$ $(36.1 \pm 0.6 \mathrm{~g} / \mathrm{dl})$, whereas, open shed in high rainfall zone recorded lower MCHC $(32.2 \pm 0.6 \mathrm{~g} / \mathrm{dl})$ content during summer. The present study differed from the findings of Randhawa et. al. (2009). They reported that $\mathrm{MCHC}$ was significantly higher during summer season compared to rainy and winter seasons.

\section{RDW}

The mean \pm S.E of RDW of animals under various housing types in Southern, Cauvery delta and High rainfall zone are presented in the Table 4. 
Table.1 Zone wise details of Mean \pm SE of WBC and RBC in various housing types during South West monsoon (SWM), North East monsoon (NEM), Winter and Summer seasons

\begin{tabular}{|c|c|c|c|c|c|c|c|c|c|c|c|c|c|c|c|c|c|c|c|c|c|c|}
\hline \multirow{4}{*}{ Name } & \multirow[t]{4}{*}{ Seasons } & \multicolumn{21}{|c|}{ Agro Climatic Zones } \\
\hline & & \multirow{2}{*}{\multicolumn{7}{|c|}{$\begin{array}{l}\text { Southern } \\
\text { Housing Type }\end{array}$}} & \multirow{2}{*}{\multicolumn{7}{|c|}{$\begin{array}{l}\text { Cauvery Delta } \\
\text { Housing Type }\end{array}$}} & \multirow{2}{*}{\multicolumn{7}{|c|}{$\begin{array}{l}\text { High Rainfall } \\
\text { Housing Type }\end{array}$}} \\
\hline & & & & & & & & & & & & & & & & & & & & & & \\
\hline & & $\begin{array}{c}\text { Thatche } \\
d\end{array}$ & Tiled & $\begin{array}{c}\text { Asbesto } \\
\mathrm{s}\end{array}$ & Metal & Open & Total & $\begin{array}{c}\text { Anov } \\
\text { a }\end{array}$ & $\begin{array}{l}\text { Thatche } \\
d\end{array}$ & Tiled & $\begin{array}{c}\text { Asbesto } \\
\mathrm{s}\end{array}$ & Metal & Open & Total & $\begin{array}{c}\text { Anov } \\
\text { a }\end{array}$ & $\begin{array}{l}\text { Thatche } \\
d\end{array}$ & Tiled & $\begin{array}{c}\text { Asbesto } \\
\mathrm{s}\end{array}$ & Metal & Open & Total & $\begin{array}{c}\text { Anov } \\
\mathrm{a}\end{array}$ \\
\hline \multirow{5}{*}{$\begin{array}{c}\text { WBC } \\
\left(10^{3} / \mu \mathrm{l}\right)\end{array}$} & SWM & $\begin{array}{r}12.3 \\
+35\end{array}$ & $\begin{array}{l}14.0 \\
+1.5\end{array}$ & $\begin{array}{c}9.6 \\
+1.3\end{array}$ & $\begin{array}{r}10.0 \\
+1.8\end{array}$ & $\begin{array}{c}9.7 \\
+0.4\end{array}$ & $\begin{array}{l}11.1 \\
+0.9\end{array}$ & \multirow[t]{2}{*}{$\begin{array}{c}\mathrm{S} \\
(.005)\end{array}$} & $\begin{array}{r}14.3 \\
+1.8\end{array}$ & $\begin{array}{l}16.0 \\
+0.9\end{array}$ & $\begin{array}{c}9.3 \\
+1.0\end{array}$ & $\begin{array}{l}10.3 \\
+1.0\end{array}$ & $\begin{array}{r}12.6 \\
\pm 1.5\end{array}$ & $\begin{array}{l}12.5 \\
+0.7\end{array}$ & \multirow[t]{2}{*}{$\begin{array}{c}\mathrm{S} \\
(.002)\end{array}$} & $\begin{array}{l}12.0 \\
\pm 0.9\end{array}$ & $\begin{array}{l}11.6 \\
+2.6\end{array}$ & $\begin{array}{l}22.8 \\
+4.7\end{array}$ & $\begin{array}{r}16.3 \\
+3.6\end{array}$ & $\begin{array}{l}13.5 \\
\pm 10\end{array}$ & $\begin{array}{l}15.2 \\
+1.4\end{array}$ & \multirow[t]{2}{*}{$\begin{array}{c}\mathrm{S} \\
(.000)\end{array}$} \\
\hline & NEM & 11.6 & 12.5 & 9.0 & 9.3 & 10.7 & 10.6 & & 13.7 & 13.8 & 9.5 & 10.7 & 12.2 & 12.0 & & 20.5 & 9.7 & 12.7 & 11.0 & 15.1 & 13.8 & \\
\hline & & \pm 1.1 & \pm 0.3 & \pm 1.1 & \pm 1.4 & \pm 0.5 & \pm 0.5 & \multirow{2}{*}{$\begin{array}{l}\mathrm{HT} \\
(.876)\end{array}$} & \pm 1.8 & \pm 1.6 & \pm 1.4 & \pm 0.8 & \pm 1.5 & \pm 0.7 & \multirow{3}{*}{$\begin{array}{c}\mathrm{HT} \\
(.002) \\
\\
\mathrm{S} * \mathrm{HT} \\
(.000)\end{array}$} & \pm 3.9 & \pm 1.4 & \pm 1.8 & \pm 1.4 & \pm 2.9 & \pm 1.2 & \multirow{3}{*}{$\begin{array}{c}\mathrm{HT} \\
(.040) \\
\\
\mathrm{S}^{*} \mathrm{HT} \\
(.023)\end{array}$} \\
\hline & Winter & $\begin{array}{c}7.3 \\
\pm 1.1\end{array}$ & $\begin{array}{c}7.0 \\
\pm 0.2\end{array}$ & $\begin{array}{c}8.2 \\
\pm 0.3\end{array}$ & $\begin{array}{c}9.7 \\
\pm 0.6\end{array}$ & $\begin{array}{l}12.1 \\
\pm 1.9\end{array}$ & $\begin{array}{c}8.8 \\
\pm 0.6\end{array}$ & & $\begin{array}{l}14.5 \\
\pm 2.0\end{array}$ & $\begin{array}{c}8.1 \\
\pm 0.9\end{array}$ & $\begin{array}{c}9.6 \\
\pm 1.0\end{array}$ & $\begin{array}{l}16.5 \\
\pm 1.4\end{array}$ & $\begin{array}{l}10.3 \\
\pm 1.7\end{array}$ & $\begin{array}{l}11.8 \\
\pm 0.8\end{array}$ & & $\begin{array}{l}14.2 \\
\pm 1.8\end{array}$ & $\begin{array}{l}12.9 \\
\pm 0.2\end{array}$ & $\begin{array}{l}15.5 \\
\pm 0.9\end{array}$ & $\begin{array}{l}12.0 \\
\pm 2.7\end{array}$ & $\begin{array}{l}12.0 \\
\pm 1.8\end{array}$ & $\begin{array}{l}13.3 \\
\pm 0.8\end{array}$ & \\
\hline & Summer & $\begin{array}{c}8.4 \\
\pm 0.5\end{array}$ & $\begin{array}{c}6.1 \\
\pm 0.8\end{array}$ & $\begin{array}{r}10.3 \\
\pm 1.2\end{array}$ & $\begin{array}{c}9.7 \\
\pm 1.2\end{array}$ & $\begin{array}{c}8.5 \\
\pm 0.8\end{array}$ & $\begin{array}{c}8.6 \\
\pm 0.5\end{array}$ & $\begin{array}{l}\mathrm{S} * \mathrm{HT} \\
(.008)\end{array}$ & $\begin{array}{c}8.9 \\
\pm 0.9\end{array}$ & $\begin{array}{c}9.1 \\
\pm 0.1\end{array}$ & $\begin{array}{c}9.0 \\
\pm 0.5\end{array}$ & $\begin{array}{l}11.1 \\
\pm 0.4\end{array}$ & $\begin{array}{c}9.7 \\
\pm 1.0\end{array}$ & $\begin{array}{c}9.6 \\
\pm 0.3\end{array}$ & & $\begin{array}{l}9.8 \\
\pm 0.5\end{array}$ & $\begin{array}{c}9.4 \\
\pm 0.9\end{array}$ & $\begin{array}{l}10.1 \\
\pm 1.4\end{array}$ & $\begin{array}{c}8.9 \\
\pm 0.7\end{array}$ & $\begin{array}{c}8.2 \\
\pm 1.2\end{array}$ & $\begin{array}{c}9.2 \\
\pm 0.4\end{array}$ & \\
\hline \multirow[t]{5}{*}{$\begin{array}{c}\mathrm{RBC} \\
\left(10^{6 / \mu l}\right)\end{array}$} & SWM & $\begin{array}{r}5.86 \\
\pm 0.21\end{array}$ & $\begin{array}{c}5.68 \\
\pm 0.18\end{array}$ & $\begin{array}{c}5.15 \\
\pm 0.46\end{array}$ & $\begin{array}{c}5.33 \\
\pm 0.36\end{array}$ & $\begin{array}{r}5.90 \\
\pm 0.21\end{array}$ & $\begin{array}{c}5.59 \\
\pm 00.14\end{array}$ & $\begin{array}{c}\mathrm{S} \\
(.001)\end{array}$ & $\begin{array}{r}5.24 \\
\pm 0.22\end{array}$ & $\begin{array}{r}5.60 \\
\pm 0.15\end{array}$ & $\begin{array}{c}5.60 \\
\pm 0.36\end{array}$ & $\begin{array}{r}5.34 \\
\pm 0.39\end{array}$ & $\begin{array}{r}5.64 \\
\pm 0.21\end{array}$ & $\begin{array}{c}5.48 \\
\pm 0.12\end{array}$ & $\begin{array}{c}\mathrm{S} \\
(.000)\end{array}$ & $\begin{array}{c}4.65 \\
\pm 0.30\end{array}$ & $\begin{array}{r}5.66 \\
\pm 1.00\end{array}$ & $\begin{array}{c}5.74 \\
\pm 0.39\end{array}$ & $\begin{array}{r}5.64 \\
\pm 0.29 \\
\end{array}$ & $\begin{array}{c}5.50 \\
\pm 0.22\end{array}$ & $\begin{array}{c}5.44 \\
\pm 0.23\end{array}$ & \multirow{3}{*}{$\begin{array}{c}\mathrm{S} \\
(.518) \\
\\
\mathrm{HT} \\
(.092)\end{array}$} \\
\hline & NEM & $\begin{array}{c}6.72 \\
\pm 1.24\end{array}$ & $\begin{array}{l}6.03 \\
\pm 0.35\end{array}$ & $\begin{array}{r}5.81 \\
\pm 0.51\end{array}$ & $\begin{array}{c}5.28 \\
\pm 0.37\end{array}$ & $\begin{array}{r}5.95 \\
\pm 0.26\end{array}$ & $\begin{array}{r}5.96 \\
\pm 0.28 \\
\end{array}$ & \multirow{4}{*}{$\begin{array}{c}\mathrm{HT} \\
(.422) \\
\mathrm{S}^{*} \mathrm{HT} \\
(.390)\end{array}$} & $\begin{array}{r}4.65 \\
\pm 0.21 \\
\end{array}$ & $\begin{array}{r}5.45 \\
\pm 0.37\end{array}$ & $\begin{array}{c}5.12 \\
\pm 0.10\end{array}$ & $\begin{array}{r}5.05 \\
\pm 0.32 \\
\end{array}$ & $\begin{array}{c}6.08 \\
\pm 0.34 \\
\end{array}$ & $\begin{array}{c}5.27 \\
\pm 0.15\end{array}$ & \multirow{4}{*}{$\begin{array}{c}\mathrm{HT} \\
(.008) \\
\mathrm{S} * \mathrm{HT} \\
(.442)\end{array}$} & $\begin{array}{c}5.18 \\
\pm 0.62\end{array}$ & $\begin{array}{r}5.28 \\
\pm 0.24\end{array}$ & $\begin{array}{r}5.66 \\
\pm 0.37\end{array}$ & $\begin{array}{r}5.35 \\
\pm 0.18\end{array}$ & $\begin{array}{c}5.53 \\
\pm 0.17\end{array}$ & $\begin{array}{r}5.40 \\
\pm 0.15\end{array}$ & \\
\hline & Winter & $\begin{array}{c}5.31 \\
\pm 0.43\end{array}$ & $\begin{array}{l}4.52 \\
\pm 0.17\end{array}$ & $\begin{array}{c}5.64 \\
\pm 0.20\end{array}$ & $\begin{array}{c}5.28 \\
\pm 0.17\end{array}$ & $\begin{array}{c}5.07 \\
\pm 0.37\end{array}$ & $\begin{array}{r}5.16 \\
\pm 0.14\end{array}$ & & $\begin{array}{l}4.32 \\
\pm 0.08\end{array}$ & $\begin{array}{r}4.39 \\
\pm 0.32\end{array}$ & $\begin{array}{c}4.75 \\
\pm 0.38\end{array}$ & $\begin{array}{l}4.65 \\
\pm 0.35\end{array}$ & $\begin{array}{c}4.79 \\
\pm 0.26\end{array}$ & $\begin{array}{c}4.58 \\
\pm 0.13\end{array}$ & & $\begin{array}{c}5.25 \\
\pm 0.46\end{array}$ & $\begin{array}{r}5.58 \\
\pm 0.33\end{array}$ & $\begin{array}{c}6.26 \\
\pm 0.31\end{array}$ & $\begin{array}{r}5.97 \\
\pm 0.27\end{array}$ & $\begin{array}{c}5.71 \\
\pm 0.55\end{array}$ & $\begin{array}{r}5.75 \\
\pm 0.18\end{array}$ & \\
\hline & Summer & $\begin{array}{c}5.22 \\
\pm 0.14\end{array}$ & $\begin{array}{l}5.07 \\
\pm 0.22\end{array}$ & $\begin{array}{r}4.49 \\
\pm 0.37\end{array}$ & $\begin{array}{c}5.48 \\
\pm 0.16\end{array}$ & $\begin{array}{l}4.71 \\
\pm 0.31\end{array}$ & $\begin{array}{l}4.99 \\
\pm 0.13\end{array}$ & & $\begin{array}{r}3.99 \\
\pm 0.27\end{array}$ & $\begin{array}{r}3.67 \\
\pm 0.13\end{array}$ & $\begin{array}{c}4.61 \\
\pm 0.51\end{array}$ & $\begin{array}{l}4.49 \\
\pm 0.10\end{array}$ & $\begin{array}{c}4.61 \\
\pm 0.14\end{array}$ & $\begin{array}{l}4.27 \\
\pm 0.13\end{array}$ & & $\begin{array}{c}5.97 \\
\pm 0.40\end{array}$ & $\begin{array}{r}5.28 \\
\pm 0.23\end{array}$ & $\begin{array}{c}6.46 \\
\pm 0.27\end{array}$ & $\begin{array}{r}5.28 \\
\pm 0.21\end{array}$ & $\begin{array}{c}4.89 \\
\pm 0.46\end{array}$ & $\begin{array}{r}5.57 \\
\pm 0.17\end{array}$ & \multirow[t]{2}{*}{$\begin{array}{l}\mathrm{S}^{*} \mathrm{HT} \\
(.575)\end{array}$} \\
\hline & Total & $\begin{array}{r}5.78 \\
\pm 0.34\end{array}$ & $\begin{array}{l}5.32 \\
\pm 0.16\end{array}$ & $\begin{array}{r}5.27 \\
\pm 0.22\end{array}$ & $\begin{array}{c}5.34 \\
\pm 0.13\end{array}$ & $\begin{array}{c}5.41 \\
\pm 0.18\end{array}$ & $\begin{array}{c}5.42 \\
\pm 0.10\end{array}$ & & $\begin{array}{l}4.55 \mathrm{e} \\
\pm 0.14\end{array}$ & $\begin{array}{l}4.77 \\
\pm 0.20\end{array}$ & $\begin{array}{c}5.02 \\
\pm 0.19\end{array}$ & $\begin{aligned} & 4.88 \\
& \pm 0.16\end{aligned}$ & $\begin{array}{r}5.28^{\mathrm{a}} \\
\pm 0.17\end{array}$ & $\begin{array}{r}4.90 \\
\pm 0.08\end{array}$ & & $\begin{array}{r}5.26 \\
\pm 0.24\end{array}$ & $\begin{array}{r}5.45 \\
\pm 0.26\end{array}$ & $\begin{array}{c}6.03 \\
\pm 0.17\end{array}$ & $\begin{array}{r}5.56 \\
\pm 0.13\end{array}$ & $\begin{array}{c}5.41 \\
\pm 0.19\end{array}$ & $\begin{array}{l}5.54 \\
\pm 0.9\end{array}$ & \\
\hline
\end{tabular}

S. Season; HT - Housing type; S*HT - Season verses Housing interaction. Figures in parenthesis indicates significance 
Table.2 Zone wise details of Mean \pm SE of $\mathrm{Hb}$ and HCT in various housing types during South West monsoon (SWM), North East monsoon (NEM), Winter and Summer seasons

\begin{tabular}{|c|c|c|c|c|c|c|c|c|c|c|c|c|c|c|c|c|c|c|c|c|c|c|}
\hline \multirow[t]{4}{*}{ Name } & \multirow[t]{4}{*}{ Seasons } & \multicolumn{21}{|c|}{ Agro Climatic Zones } \\
\hline & & \multirow{2}{*}{\multicolumn{7}{|c|}{$\begin{array}{c}\text { Southern } \\
\text { Housing Type }\end{array}$}} & \multirow{2}{*}{\multicolumn{7}{|c|}{$\begin{array}{l}\text { Cauvery Delta } \\
\text { Housing Type }\end{array}$}} & \multirow{2}{*}{\multicolumn{7}{|c|}{$\begin{array}{l}\text { High Rainfall } \\
\text { Housing Type }\end{array}$}} \\
\hline & & & & & & & & & & & & & & & & & & & & & & \\
\hline & & Thatched & Tiled & Asbestos & Metal & Open & Total & Anova & Thatched & Tiled & Asbestos & Metal & Open & Total & Anova & Thatched & Tiled & Asbestos & Metal & Open & Total & Anova \\
\hline \multirow[t]{5}{*}{$\begin{array}{c}\mathrm{Hb} \\
(\mathrm{g} / \mathrm{dl})\end{array}$} & SWM & $\begin{array}{c}9.7 \\
\pm 0.1\end{array}$ & $\begin{array}{c}8.9 \\
\pm 0.4\end{array}$ & $\begin{array}{c}8.9 \\
\pm 0.8\end{array}$ & $\begin{array}{c}8.1 \\
\pm 0.8\end{array}$ & $\begin{array}{c}9.4 \\
\pm 0.4\end{array}$ & $\begin{array}{r}9.0 \\
\pm 0.3\end{array}$ & \multirow{2}{*}{$\begin{array}{c}\mathrm{S} \\
(.000) \\
\mathrm{HT} \\
(.209)\end{array}$} & $\begin{array}{c}8.8 \\
\pm 0.4\end{array}$ & $\begin{array}{c}8.1 \\
\pm 0.3\end{array}$ & $\begin{array}{c}8.5 \\
\pm 1.0\end{array}$ & $\begin{array}{c}8.4 \\
\pm 0.5\end{array}$ & $\begin{array}{c}9.1 \\
\pm 0.4\end{array}$ & $\begin{array}{c}8.6 \\
\pm 0.2\end{array}$ & \multirow{2}{*}{$\begin{array}{c}\mathrm{S} \\
(.000) \\
\\
\mathrm{HT} \\
(.006)\end{array}$} & $\begin{array}{c}8.4 \\
\pm 0.5\end{array}$ & $\begin{array}{c}9.1 \\
\pm 1.9\end{array}$ & $\begin{array}{c}9.5 \\
\pm 0.6\end{array}$ & $\begin{array}{c}9.9 \\
\pm 0.4\end{array}$ & $\begin{array}{c}9.1 \\
\pm 0.3\end{array}$ & $\begin{array}{r}9.2 \\
\pm 0.4\end{array}$ & \multirow{2}{*}{$\begin{array}{c}\mathrm{S} \\
(.177) \\
\\
\mathrm{HT} \\
(.708)\end{array}$} \\
\hline & NEM & $\begin{array}{c}9.9 \\
\pm 0.7\end{array}$ & $\begin{array}{r}9.3 \\
\pm 0.3\end{array}$ & $\begin{array}{c}9.5 \\
\pm 0.8\end{array}$ & $\begin{array}{c}8.2 \\
\pm 0.7\end{array}$ & $\begin{array}{c}9.2 \\
\pm 0.4\end{array}$ & $\begin{array}{c}9.2 \\
\pm 0.3\end{array}$ & & $\begin{array}{c}8.3 \\
\pm 0.2\end{array}$ & $\begin{array}{c}8.0 \\
\pm 0.5\end{array}$ & $\begin{array}{c}7.5 \\
\pm 0.7\end{array}$ & $\begin{array}{c}7.3 \\
\pm 0.4\end{array}$ & $\begin{array}{c}9.0 \\
\pm 0.1\end{array}$ & $\begin{array}{c}8.0 \\
\pm 0.2\end{array}$ & & $\begin{array}{c}8.0 \\
\pm 0.8\end{array}$ & $\begin{array}{c}8.6 \\
\pm 0.4\end{array}$ & $\begin{array}{c}9.5 \\
\pm 0.6\end{array}$ & $\begin{array}{c}8.7 \\
\pm 0.6\end{array}$ & $\begin{array}{c}9.0 \\
\pm 0.3\end{array}$ & $\begin{array}{c}8.7 \\
\pm 0.3\end{array}$ & \\
\hline & Winter & $\begin{array}{l}7.9 \\
\pm 0.7\end{array}$ & $\begin{array}{c}6.6 \\
\pm 0.2\end{array}$ & $\begin{array}{c}7.9 \\
\pm 0.2\end{array}$ & $\begin{array}{l}8.6 \\
\pm 0.3\end{array}$ & $\begin{array}{c}7.7 \\
\pm 0.6\end{array}$ & $\begin{array}{r}7.7 \\
\pm 0.2\end{array}$ & \multirow[t]{3}{*}{$(.068)$} & $\begin{array}{l}7.4 \\
\pm 0.3\end{array}$ & $\begin{array}{c}6.4 \\
\pm 0.3\end{array}$ & $\begin{array}{c}7.3 \\
\pm 0.8\end{array}$ & $\begin{array}{c}6.7 \\
\pm 0.4\end{array}$ & $\begin{array}{r}7.6 \\
\pm 0.2\end{array}$ & $\begin{array}{r}7.1 \\
\pm 0.2\end{array}$ & \multirow[t]{3}{*}{$\begin{array}{l}\mathrm{S} * \mathrm{HT} \\
(.759)\end{array}$} & $\begin{array}{c}9.3 \\
\pm 0.6\end{array}$ & $\begin{array}{l}8.7 \\
\pm 0.5\end{array}$ & $\begin{array}{c}8.8 \\
\pm 0.4\end{array}$ & $\begin{array}{l}9.8 \\
\pm 0.3\end{array}$ & $\begin{array}{r}9.3 \\
\pm 1.1\end{array}$ & $\begin{array}{c}9.2 \\
\pm 0.3\end{array}$ & \multirow[t]{3}{*}{$\begin{array}{l}\mathrm{S} * \mathrm{HT} \\
(.562)\end{array}$} \\
\hline & Summer & $7.9 \pm 0.2$ & $\begin{array}{l}7.2 \\
\pm 0.5\end{array}$ & $\begin{array}{c}6.6 \\
\pm 0.5\end{array}$ & $\begin{array}{c}8.3 \\
\pm 0.3\end{array}$ & $\begin{array}{c}7.1 \\
\pm 0.4\end{array}$ & $\begin{array}{c}7.4 \\
\pm 0.2\end{array}$ & & $\begin{array}{c}6.0 \\
\pm 0.4\end{array}$ & $\begin{array}{c}5.2 \\
\pm 0.2\end{array}$ & $\begin{array}{c}6.9 \\
\pm 0.8\end{array}$ & $\begin{array}{c}6.2 \\
\pm 0.1\end{array}$ & $\begin{array}{c}6.8 \\
\pm 0.2\end{array}$ & $\begin{array}{c}6.2 \\
\pm 0.2\end{array}$ & & $\begin{array}{c}9.3 \\
\pm 0.5\end{array}$ & $\begin{array}{r}8.0 \\
\pm 0.4\end{array}$ & $\begin{array}{c}9.2 \\
\pm 0.7\end{array}$ & $\begin{array}{c}7.7 \\
\pm 0.4\end{array}$ & $\begin{array}{r}7.4 \\
\pm 0.6\end{array}$ & $\begin{array}{r}8.3 \\
\pm 0.3\end{array}$ & \\
\hline & Total & $\begin{array}{c}8.9 \\
\pm 0.3\end{array}$ & $\begin{array}{l}8.0 \\
\pm 0.3\end{array}$ & $\begin{array}{c}8.2 \\
\pm 0.4\end{array}$ & $\begin{array}{c}8.3 \\
\pm 0.3\end{array}$ & $\begin{array}{r}8.3 \\
\pm 0.3\end{array}$ & $\begin{array}{c}8.3 \\
\pm 0.1\end{array}$ & & $\begin{array}{c}7.6 \\
\pm 0.3\end{array}$ & $\begin{array}{c}6.9 \\
\pm 0.3\end{array}$ & $\begin{array}{c}7.5 \\
\pm 0.4\end{array}$ & $\begin{array}{r}7.2 \\
\pm 0.2\end{array}$ & $\begin{array}{l}8.1 \mathrm{~d} \\
\pm 0.2\end{array}$ & $\begin{array}{c}7.5 \\
\pm 0.1\end{array}$ & & $\begin{array}{c}8.7 \\
\pm 0.3\end{array}$ & $\begin{array}{l}8.6 \\
\pm 0.5\end{array}$ & $\begin{array}{c}9.2 \\
\pm 0.3\end{array}$ & $\begin{array}{c}9.0 \\
\pm 0.3\end{array}$ & $\begin{array}{r}8.7 \\
\pm 0.3\end{array}$ & $\begin{array}{r}8.9 \\
\pm 0.2\end{array}$ & \\
\hline \multirow[t]{5}{*}{$\begin{array}{l}\text { HCT } \\
(\%)\end{array}$} & SWM & $\begin{array}{l}27.3 \\
\pm 0.3\end{array}$ & $\begin{array}{l}25.2 \\
\pm 1.1\end{array}$ & $\begin{array}{l}25.0 \\
\pm 2.3\end{array}$ & $\begin{array}{l}23.5 \\
\pm 2.0\end{array}$ & $\begin{array}{l}27.5 \\
\pm 1.0\end{array}$ & $\begin{array}{l}25.7 \\
\pm 0.7\end{array}$ & \multirow{5}{*}{$\begin{array}{c}\mathrm{S} \\
(.000) \\
\mathrm{HT} \\
(.803) \\
\\
\mathrm{S} * \mathrm{HT} \\
(.186)\end{array}$} & $\begin{array}{l}24.5 \\
\pm 1.0\end{array}$ & $\begin{array}{l}23.7 \\
\pm 0.8\end{array}$ & $\begin{array}{l}25.0 \\
\pm 1.9\end{array}$ & $\begin{array}{l}24.6 \\
\pm 1.3\end{array}$ & $\begin{array}{l}24.6 \\
\pm 0.8\end{array}$ & $\begin{array}{l}24.5 \\
\pm 0.5\end{array}$ & \multirow{5}{*}{$\begin{array}{c}\mathrm{S} \\
(.000) \\
\\
\mathrm{HT} \\
(.029) \\
\\
\mathrm{S}^{*} \mathrm{HT} \\
(.690)\end{array}$} & $\begin{array}{l}24.5 \\
\pm 1.3\end{array}$ & $\begin{array}{l}26.4 \\
\pm 5.0\end{array}$ & $\begin{array}{l}26.3 \\
\pm 1.8\end{array}$ & $\begin{array}{l}27.9 \\
\pm 1.2\end{array}$ & $\begin{array}{l}25.8 \\
\pm 0.9\end{array}$ & $\begin{array}{l}26.2 \\
\pm 1.1\end{array}$ & \multirow{5}{*}{$\begin{array}{c}\mathrm{S} \\
(.378) \\
\\
\mathrm{HT} \\
(.838) \\
\mathrm{S} \\
\mathrm{S} \cdot \mathrm{HT} \\
(.723)\end{array}$} \\
\hline & NEM & $\begin{array}{l}25.4 \\
\pm 2.7\end{array}$ & $\begin{array}{l}27.5 \\
\pm 0.6\end{array}$ & $\begin{array}{l}27.8 \\
\pm 2.1\end{array}$ & $\begin{array}{l}25.2 \\
\pm 0.9\end{array}$ & $\begin{array}{l}28.0 \\
\pm 1.0\end{array}$ & $\begin{array}{l}26.8 \\
\pm 0.7\end{array}$ & & $\begin{array}{l}24.6 \\
\pm 0.6\end{array}$ & $\begin{array}{l}23.5 \\
\pm 0.8\end{array}$ & $\begin{array}{l}24.8 \\
\pm 2.0\end{array}$ & $\begin{array}{l}24.4 \\
\pm 1.6\end{array}$ & $\begin{array}{l}25.1 \\
\pm 0.7\end{array}$ & $\begin{array}{l}24.5 \\
\pm 0.5\end{array}$ & & $\begin{array}{l}23.4 \\
\pm 2.4\end{array}$ & $\begin{array}{l}25.7 \\
\pm 1.3\end{array}$ & $\begin{array}{l}27.8 \\
\pm 1.6\end{array}$ & $\begin{array}{l}26.5 \\
\pm 1.2\end{array}$ & $\begin{array}{l}25.7 \\
\pm 0.5\end{array}$ & $\begin{array}{l}25.8 \\
\pm 0.7\end{array}$ & \\
\hline & Winter & $\begin{array}{l}23.4 \\
\pm 1.9\end{array}$ & $\begin{array}{l}21.9 \\
\pm 0.5\end{array}$ & $\begin{array}{l}25.1 \\
\pm 1.8\end{array}$ & $\begin{array}{l}24.1 \\
\pm 1.0\end{array}$ & $\begin{array}{l}24.3 \\
\pm 1.8\end{array}$ & $\begin{array}{l}23.7 \\
\pm 0.7\end{array}$ & & $\begin{array}{l}22.6 \\
\pm 0.7\end{array}$ & $\begin{array}{l}19.5 \\
\pm 0.9\end{array}$ & $\begin{array}{r}19.9 \\
\pm 3.0\end{array}$ & $\begin{array}{l}22.5 \\
\pm 1.3\end{array}$ & $\begin{array}{l}23.3 \\
\pm 0.4\end{array}$ & $\begin{array}{l}21.6 \\
\pm 0.7\end{array}$ & & $\begin{array}{l}27.8 \\
\pm 1.5\end{array}$ & $\begin{array}{l}25.4 \\
\pm 1.2\end{array}$ & $\begin{array}{l}25.7 \\
\pm 1.5\end{array}$ & $\begin{array}{l}27.9 \\
\pm 0.9\end{array}$ & $\begin{array}{r}27.0 \\
\pm 2.9\end{array}$ & $\begin{array}{l}26.8 \\
\pm 0.7\end{array}$ & \\
\hline & Summer & $\begin{array}{l}24.0 \\
\pm 0.7\end{array}$ & $\begin{array}{r}22.0 \\
\pm 1.6\end{array}$ & $\begin{array}{r}19.9 \\
\pm 1.2\end{array}$ & $\begin{array}{l}24.6 \\
\pm 0.5\end{array}$ & $\begin{array}{l}21.3 \\
\pm 1.3\end{array}$ & $\begin{array}{r}22.4 \\
\pm 0.6\end{array}$ & & $\begin{array}{r}18.2 \\
\pm 1.2\end{array}$ & $\begin{array}{l}15.5 \\
\pm 0.1\end{array}$ & $\begin{array}{r}20.8 \\
\pm 2.4\end{array}$ & $\begin{array}{l}19.1 \\
\pm 0.2\end{array}$ & $\begin{array}{l}21.3 \\
\pm 0.5\end{array}$ & $\begin{array}{r}19.0 \\
\pm 0.6\end{array}$ & & $\begin{array}{l}27.2 \\
\pm 1.5\end{array}$ & $\begin{array}{l}23.9 \\
\pm 0.9\end{array}$ & $\begin{array}{r}26.3 \\
\pm 2.1\end{array}$ & $\begin{array}{l}23.3 \\
\pm 0.8\end{array}$ & $\begin{array}{r}23.0 \\
\pm 1.8\end{array}$ & $\begin{array}{l}24.7 \\
\pm 0.7\end{array}$ & \\
\hline & Total & $\begin{array}{l}25.0 \\
\pm 0.9\end{array}$ & $\begin{array}{l}24.1 \\
\pm 0.7\end{array}$ & $\begin{array}{l}24.4 \\
\pm 1.1\end{array}$ & $\begin{array}{l}24.4 \\
\pm 0.6\end{array}$ & $\begin{array}{l}25.3 \\
\pm 0.8\end{array}$ & $\begin{array}{l}24.6 \\
\pm 0.4\end{array}$ & & $\begin{array}{l}22.5 \\
\pm 0.7\end{array}$ & $\begin{array}{c}20.5 \mathrm{e} \\
\pm 0.8\end{array}$ & $\begin{array}{l}22.6 \\
\pm 1.2\end{array}$ & $\begin{array}{l}22.6 \\
\pm 0.7\end{array}$ & $\begin{array}{c}23.6 \mathrm{~b} \\
\pm 0.4\end{array}$ & $\begin{array}{l}22.4 \\
\pm 0.4\end{array}$ & & $\begin{array}{l}25.8 \\
\pm 0.9\end{array}$ & $\begin{array}{l}25.3 \\
\pm 1.3\end{array}$ & $\begin{array}{r}26.5 \\
\pm 0.8\end{array}$ & $\begin{array}{l}26.4 \\
\pm 0.6\end{array}$ & $\begin{array}{l}25.4 \\
\pm 0.9\end{array}$ & $\begin{array}{r}25.9 \\
\pm 0.4\end{array}$ & \\
\hline
\end{tabular}

S. Season; HT - Housing type; S*HT - Season verses Housing interaction. Figures in parenthesis indicates significance 
Table.3 Zone wise details of Mean \pm SE of MCV and MCH in various housing types during South West monsoon (SWM), North East monsoon (NEM), Winter and Summer seasons

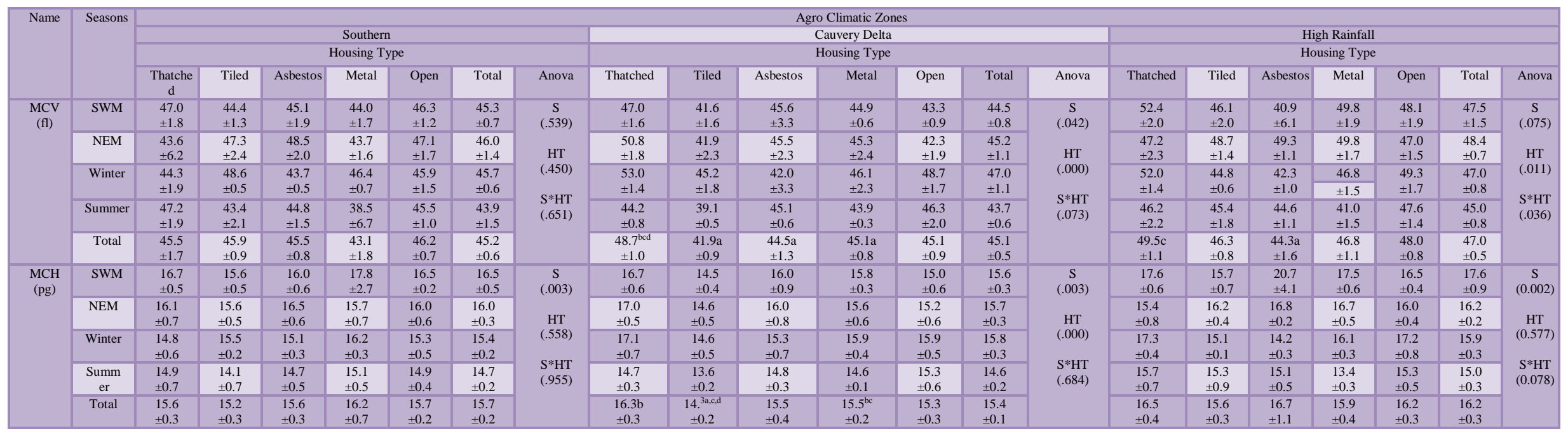

S. Season; HT - Housing type; $\mathrm{S} * \mathrm{HT}-$ Season verses Housing interaction. Figures in parenthesis indicates significance 
Table.4 Zone wise details of Mean \pm SE of MCHC and RDW in various housing types during South West monsoon (SWM), North East monsoon (NEM), Winter and Summer seasons

\begin{tabular}{|c|c|c|c|c|c|c|c|c|c|c|c|c|c|c|c|c|c|c|c|c|c|c|}
\hline \multirow[t]{4}{*}{ Name } & \multirow[t]{4}{*}{ Seasons } & \multicolumn{21}{|c|}{ Agro Climatic Zones } \\
\hline & & \multirow{2}{*}{\multicolumn{7}{|c|}{$\begin{array}{c}\text { Southern } \\
\text { Housing Type }\end{array}$}} & \multirow{2}{*}{\multicolumn{7}{|c|}{$\begin{array}{l}\text { Cauvery Delta } \\
\text { Housing Type }\end{array}$}} & \multirow{2}{*}{\multicolumn{7}{|c|}{$\begin{array}{l}\text { High Rainfall } \\
\text { Housing Type }\end{array}$}} \\
\hline & & & & & & & & & & & & & & & & & & & & & & \\
\hline & & Thatched & Tiled & Asbestos & Metal & Open & Total & Anova & Thatched & Tiled & Asbestos & Metal & Open & Total & Anova & Thatched & Tiled & Asbestos & Metal & Open & Total & Anova \\
\hline \multirow[t]{5}{*}{$\begin{array}{l}\text { MCHC } \\
\text { (g/dl) }\end{array}$} & SWM & $\begin{array}{l}35.6 \\
\pm 0.3\end{array}$ & $\begin{array}{l}35.4 \\
\pm 0.5\end{array}$ & $\begin{array}{l}36.1 \\
\pm 0.6\end{array}$ & $\begin{array}{l}34.2 \\
\pm 0.7\end{array}$ & $\begin{array}{l}35.5 \\
\pm 0.8\end{array}$ & $\begin{array}{l}35.4 \\
\pm 0.3\end{array}$ & \multirow{5}{*}{$\begin{array}{c}\mathrm{S} \\
(.003) \\
\\
\mathrm{HT} \\
(.558) \\
\mathrm{S}^{*} \mathrm{HT} \\
(.955)\end{array}$} & $\begin{array}{l}35.6 \\
\pm 0.2\end{array}$ & $\begin{array}{l}34.7 \\
\pm 0.2\end{array}$ & $\begin{array}{l}35.3 \\
\pm 0.7\end{array}$ & $\begin{array}{l}34.0 \\
\pm 0.2\end{array}$ & $\begin{array}{l}36.0 \\
\pm 0.5\end{array}$ & $\begin{array}{l}35.1 \\
\pm 0.2\end{array}$ & \multirow{5}{*}{$\begin{array}{c}\mathrm{S} \\
(.003) \\
\mathrm{HT} \\
(.000) \\
\mathrm{S}^{*} \mathrm{HT} \\
(.684)\end{array}$} & $\begin{array}{l}34.4 \\
\pm 0.3\end{array}$ & $\begin{array}{l}34.2 \\
\pm 1.0\end{array}$ & $\begin{array}{l}36.0 \\
\pm 0.5\end{array}$ & $\begin{array}{l}35.3 \\
\pm 0.4\end{array}$ & $\begin{array}{l}35.3 \\
\pm 0.3\end{array}$ & $\begin{array}{l}35.0 \\
\pm 0.3\end{array}$ & \multirow{5}{*}{$\begin{array}{c}\mathrm{S} \\
(.002) \\
\\
\mathrm{HT} \\
(.577) \\
\mathrm{S} * \mathrm{HT} \\
(.078)\end{array}$} \\
\hline & NEM & $\begin{array}{l}34.0 \\
\pm 0.3\end{array}$ & $\begin{array}{l}34.5 \\
\pm 0.7\end{array}$ & $\begin{array}{l}34.1 \\
\pm 0.4\end{array}$ & $\begin{array}{l}34.6 \\
\pm 0.6\end{array}$ & $\begin{array}{l}33.8 \\
\pm 0.1\end{array}$ & $\begin{array}{l}34.2 \\
\pm 0.2\end{array}$ & & $\begin{array}{l}33.2 \\
\pm 0.5\end{array}$ & $\begin{array}{l}34.2 \\
\pm 1.0\end{array}$ & $\begin{array}{l}34.8 \\
\pm 0.9\end{array}$ & $\begin{array}{l}33.4 \\
\pm 0.5\end{array}$ & $\begin{array}{l}36.1 \\
\pm 0.6\end{array}$ & $\begin{array}{l}34.3 \\
\pm 0.4\end{array}$ & & $\begin{array}{l}32.6 \\
\pm 0.3\end{array}$ & $\begin{array}{r}33.5 \\
\pm 0.2\end{array}$ & $\begin{array}{l}34.3 \\
\pm 0.6\end{array}$ & $\begin{array}{l}33.5 \\
\pm 0.5\end{array}$ & $\begin{array}{l}34.3 \\
\pm 0.5\end{array}$ & $\begin{array}{l}33.6 \\
\pm 0.2\end{array}$ & \\
\hline & Winter & $\begin{array}{l}31.9 \\
\pm 1.9\end{array}$ & $\begin{array}{l}32.4 \\
\pm 0.3\end{array}$ & $\begin{array}{l}34.5 \\
\pm 0.5\end{array}$ & $\begin{array}{l}35.0 \\
\pm 0.4\end{array}$ & $\begin{array}{l}33.5 \\
\pm 0.5\end{array}$ & $\begin{array}{l}33.4 \\
\pm 0.4\end{array}$ & & $\begin{array}{r}32.9 \\
\pm 0.4\end{array}$ & $\begin{array}{l}32.9 \\
\pm 0.1\end{array}$ & $\begin{array}{l}33.8 \\
\pm 0.5\end{array}$ & $\begin{array}{l}32.9 \\
\pm 0.5\end{array}$ & $\begin{array}{l}33.0 \\
\pm 0.6\end{array}$ & $\begin{array}{l}33.1 \\
\pm 0.2\end{array}$ & & $\begin{array}{l}33.5 \\
\pm 0.3\end{array}$ & $\begin{array}{l}34.1 \\
\pm 0.6\end{array}$ & $\begin{array}{r}34.3 \\
\pm 1.2\end{array}$ & $\begin{array}{r}34.5 \\
\pm 0.4\end{array}$ & $\begin{array}{l}34.8 \\
\pm 0.4\end{array}$ & $\begin{array}{l}34.2 \\
\pm 0.3\end{array}$ & \\
\hline & Summer & $\begin{array}{l}33.2 \\
\pm 0.1\end{array}$ & $\begin{array}{l}32.8 \\
\pm 0.1\end{array}$ & $\begin{array}{l}33.1 \\
\pm 0.5\end{array}$ & $\begin{array}{l}34.3 \\
\pm 1.1\end{array}$ & $\begin{array}{l}32.6 \\
\pm 0.2\end{array}$ & $\begin{array}{l}33.2 \\
\pm 0.3\end{array}$ & & $\begin{array}{l}32.8 \\
\pm 0.4\end{array}$ & $\begin{array}{l}34.5 \\
\pm 0.5\end{array}$ & $\begin{array}{l}32.9 \\
\pm 0.4\end{array}$ & $\begin{array}{l}32.8 \\
\pm 0.4\end{array}$ & $\begin{array}{l}33.6 \\
\pm 0.5\end{array}$ & $\begin{array}{l}33.3 \\
\pm 0.2\end{array}$ & & $\begin{array}{l}34.2 \\
\pm 0.4\end{array}$ & $\begin{array}{l}33.6 \\
\pm 0.6\end{array}$ & $\begin{array}{r}33.9 \\
\pm 0.3\end{array}$ & $\begin{array}{r}32.9 \\
\pm 0.4\end{array}$ & $\begin{array}{l}32.2 \\
\pm 0.6\end{array}$ & $\begin{array}{l}33.3 \\
\pm 0.2\end{array}$ & \\
\hline & Total & $\begin{array}{l}33.7 \\
\pm 0.5\end{array}$ & $\begin{array}{l}33.7 \\
\pm 0.3\end{array}$ & $\begin{array}{l}34.4 \\
\pm 0.3\end{array}$ & $\begin{array}{l}34.5 \\
\pm 0.4\end{array}$ & $\begin{array}{l}33.8 \\
\pm 0.3\end{array}$ & $\begin{array}{l}34.0 \\
\pm 0.2\end{array}$ & & $\begin{array}{l}33.6 \\
\pm 0.3\end{array}$ & $\begin{array}{l}34.1 \\
\pm 0.3\end{array}$ & $\begin{array}{l}34.2 \\
\pm 0.4\end{array}$ & $\begin{array}{l}33.3 \\
\pm 0.2\end{array}$ & $\begin{array}{l}34.7 \\
\pm 0.4\end{array}$ & $\begin{array}{l}34.0 \\
\pm 0.1\end{array}$ & & $\begin{array}{l}33.7 \\
\pm 0.2\end{array}$ & $\begin{array}{l}33.8 \\
\pm 0.3\end{array}$ & $\begin{array}{l}34.6 \\
\pm 0.4\end{array}$ & $\begin{array}{l}34.0 \\
\pm 0.3\end{array}$ & $\begin{array}{l}34.1 \\
\pm 0.3\end{array}$ & $\begin{array}{l}34.1 \\
\pm 0.1\end{array}$ & \\
\hline \multirow[t]{6}{*}{$\begin{array}{l}\text { RDW } \\
(\%)\end{array}$} & SWM & $\begin{array}{l}17.7 \\
\pm 0.5\end{array}$ & $\begin{array}{l}16.6 \\
\pm 0.5\end{array}$ & $\begin{array}{l}18.7 \\
\pm 0.5\end{array}$ & $\begin{array}{l}17.0 \\
\pm 0.3\end{array}$ & $\begin{array}{l}16.6 \\
\pm 0.5\end{array}$ & $\begin{array}{l}17.3 \\
\pm 0.2\end{array}$ & \multirow{3}{*}{$\begin{array}{c}\mathrm{S} \\
(.008) \\
\mathrm{HT} \\
(.065)\end{array}$} & $\begin{array}{l}18.2 \\
\pm 0.4\end{array}$ & $\begin{array}{l}16.7 \\
\pm 0.5\end{array}$ & $\begin{array}{l}18.0 \\
\pm 0.5\end{array}$ & $\begin{array}{l}17.4 \\
\pm 0.2\end{array}$ & $\begin{array}{l}17.1 \\
\pm 0.8\end{array}$ & $\begin{array}{l}17.5 \\
\pm 0.2\end{array}$ & $\begin{array}{c}\mathrm{S} \\
(.989)\end{array}$ & $\begin{array}{l}17.7 \\
\pm 0.9\end{array}$ & $\begin{array}{l}17.7 \\
\pm 0.4\end{array}$ & $\begin{array}{l}16.7 \\
\pm 0.3\end{array}$ & $\begin{array}{l}16.8 \\
\pm 0.5\end{array}$ & $\begin{array}{l}16.6 \\
\pm 0.3\end{array}$ & $\begin{array}{l}17.1 \\
\pm 0.2\end{array}$ & \multirow[t]{6}{*}{$\begin{array}{c}\mathrm{S} \\
(.497)\end{array}$} \\
\hline & NEM & $\begin{array}{l}16.6 \\
\pm 0.2\end{array}$ & $\begin{array}{l}16.2 \\
\pm 0.5\end{array}$ & $\begin{array}{l}17.0 \\
\pm 0.3\end{array}$ & $\begin{array}{l}17.2 \\
\pm 0.3\end{array}$ & $\begin{array}{l}16.6 \\
\pm 0.6\end{array}$ & $\begin{array}{l}16.7 \\
\pm 0.2\end{array}$ & & $\begin{array}{l}17.5 \\
\pm 0.5\end{array}$ & $\begin{array}{l}17.0 \\
\pm 0.5\end{array}$ & $\begin{array}{l}17.8 \\
\pm 0.5\end{array}$ & $\begin{array}{l}17.5 \\
\pm 0.2\end{array}$ & $\begin{array}{l}17.1 \\
\pm 0.5\end{array}$ & $\begin{array}{l}17.3 \\
\pm 0.2\end{array}$ & HT & $\begin{array}{l}18.4 \\
\pm 0.7\end{array}$ & $\begin{array}{l}17.0 \\
\pm 0.5\end{array}$ & $\begin{array}{l}16.8 \\
\pm 0.3\end{array}$ & $\begin{array}{l}17.1 \\
\pm 0.2\end{array}$ & $\begin{array}{l}17.6 \\
\pm 0.4\end{array}$ & $\begin{array}{l}17.4 \\
\pm 0.2\end{array}$ & \\
\hline & Winter & $\begin{array}{l}16.8 \\
\pm 0.3\end{array}$ & $\begin{array}{l}18.0 \\
\pm 0.3\end{array}$ & $\begin{array}{l}16.6 \\
\pm 0.2\end{array}$ & $\begin{array}{l}17.6 \\
\pm 0.4\end{array}$ & $\begin{array}{l}17.8 \\
\pm 0.4\end{array}$ & $\begin{array}{l}17.4 \\
\pm 0.2\end{array}$ & & $\begin{array}{l}16.6 \\
\pm 0.2\end{array}$ & $\begin{array}{l}17.5 \\
\pm 0.2\end{array}$ & $\begin{array}{l}17.6 \\
\pm 0.2\end{array}$ & $\begin{array}{l}17.7 \\
\pm 0.4\end{array}$ & $\begin{array}{l}17.4 \\
\pm 0.2\end{array}$ & $\begin{array}{l}17.4 \\
\pm 0.1\end{array}$ & \multirow{4}{*}{$\begin{array}{l}\mathrm{S}^{*} \mathrm{HT} \\
(.165)\end{array}$} & $\begin{array}{l}16.9 \\
\pm 0.4\end{array}$ & $\begin{array}{l}16.6 \\
\pm 0.2\end{array}$ & $\begin{array}{l}18.3 \\
\pm 0.3\end{array}$ & $\begin{array}{l}16.4 \\
\pm 0.2\end{array}$ & $\begin{array}{l}16.9 \\
\pm 0.4\end{array}$ & $\begin{array}{l}17.0 \\
\pm 0.2\end{array}$ & \\
\hline & Summe & 16.6 & 17.5 & 18.3 & 17.7 & 17.5 & 17.5 & \multirow{3}{*}{$\begin{array}{l}S^{* * \mathrm{HT}} \\
(.001)\end{array}$} & 18.2 & 18.3 & 17.5 & 17.3 & 15.6 & 17.4 & & 17.0 & 17.1 & 17.1 & 17.5 & 16.6 & 17.0 & \\
\hline & $\mathrm{r}$ & \pm 0.1 & \pm 0.3 & \pm 0.4 & \pm 0.3 & \pm 0.2 & \pm 0.2 & & \pm 0.3 & \pm 0.4 & \pm 0.7 & \pm 0.2 & \pm 1.7 & \pm 0.4 & & \pm 0.2 & \pm 0.4 & \pm 0.3 & \pm 0.2 & \pm 0.3 & \pm 0.1 & \\
\hline & Total & $\begin{array}{l}16.9 \mathrm{c} \\
\pm 0.2\end{array}$ & $\begin{array}{l}17.1 \\
\pm 0.2\end{array}$ & $\begin{array}{l}17.6 \mathrm{a} \\
\pm 0.3\end{array}$ & $\begin{array}{l}17.4 \\
\pm 0.2\end{array}$ & $\begin{array}{l}17.2 \\
\pm 0.2\end{array}$ & $\begin{array}{l}17.2 \\
\pm 0.1\end{array}$ & & $\begin{array}{l}17.6 \\
\pm 0.2\end{array}$ & $\begin{array}{l}17.4 \\
\pm 0.2\end{array}$ & $\begin{array}{l}17.7 \\
\pm 0.2\end{array}$ & $\begin{array}{l}17.5 \\
\pm 0.1\end{array}$ & $\begin{array}{l}16.8 \\
\pm 0.5\end{array}$ & $\begin{array}{l}17.4 \\
\pm 0.1\end{array}$ & & $\begin{array}{l}17.5 \\
\pm 0.3\end{array}$ & $\begin{array}{l}17.1 \\
\pm 0.2\end{array}$ & $\begin{array}{l}17.2 \\
\pm 0.2\end{array}$ & $\begin{array}{l}16.9 \\
\pm 0.2\end{array}$ & $\begin{array}{l}16.9 \\
\pm 0.2\end{array}$ & $\begin{array}{l}17.1 \\
\pm 0.1\end{array}$ & \\
\hline
\end{tabular}

S. Season; HT - Housing type; S*HT - Season verses Housing interaction. Figures in parenthesis indicates significance 
Table.5 Zone wise details of Mean \pm SE of PLT and MPV in various housing types during South West monsoon (SWM), North East monsoon (NEM), Winter and Summer seasons

\begin{tabular}{|c|c|c|c|c|c|c|c|c|c|c|c|c|c|c|c|c|c|c|c|c|c|c|}
\hline \multirow[t]{4}{*}{ Name } & \multirow[t]{4}{*}{ Seasons } & \multicolumn{21}{|c|}{ Agro Climatic Zones } \\
\hline & & \multirow{2}{*}{\multicolumn{7}{|c|}{$\begin{array}{c}\text { Southern } \\
\text { Housing Type }\end{array}$}} & \multirow{2}{*}{\multicolumn{7}{|c|}{\begin{tabular}{|l|} 
Cauvery Delta \\
Housing Type
\end{tabular}}} & \multirow{2}{*}{\multicolumn{7}{|c|}{$\begin{array}{l}\text { High Rainfall } \\
\text { Housing Type }\end{array}$}} \\
\hline & & & & & & & & & & & & & & & & & & & & & & \\
\hline & & $\begin{array}{l}\text { Thatche } \\
\text { d }\end{array}$ & Tiled & Asbestos & Metal & Open & Total & Anova & Thatched & Tiled & Asbestos & Metal & Open & Total & Anova & Thatched & Tiled & Asbestos & Metal & Open & Total & Anova \\
\hline \multirow[t]{8}{*}{$\begin{array}{c}\text { PLT } \\
\left(10^{3} / \mathrm{Ml}\right)\end{array}$} & SWM & $\begin{array}{l}105 \\
\pm 17\end{array}$ & $\begin{array}{l}146 \\
\pm 31\end{array}$ & $\begin{array}{l}254 \\
\pm 50\end{array}$ & $\begin{array}{l}147 \\
\pm 45\end{array}$ & $\begin{array}{l}251 \\
\pm 49\end{array}$ & $\begin{array}{l}181 \\
\pm 20\end{array}$ & \multirow{3}{*}{$\begin{array}{c}\mathrm{S} \\
(0.000)\end{array}$} & $\begin{array}{l}173 \\
\pm 21\end{array}$ & $\begin{array}{l}238 \\
\pm 73\end{array}$ & $\begin{array}{l}259 \\
\pm 53\end{array}$ & $\begin{array}{l}212 \\
\pm 41\end{array}$ & $\begin{array}{l}242 \\
\pm 52\end{array}$ & $\begin{array}{l}225 \\
\pm 22\end{array}$ & \multirow{2}{*}{$\begin{array}{c}\mathrm{S} \\
(0.029)\end{array}$} & $\begin{array}{l}200 \\
\pm 49\end{array}$ & $\begin{array}{l}146 \\
\pm 31\end{array}$ & $\begin{array}{l}182 \\
\pm 26\end{array}$ & $\begin{array}{l}193 \\
\pm 25\end{array}$ & $\begin{array}{l}160 \\
\pm 19\end{array}$ & $\begin{array}{l}176 \\
\pm 14\end{array}$ & \multirow{2}{*}{$\begin{array}{c}\mathrm{S} \\
(0.000\end{array}$} \\
\hline & NEM & 305 & 238 & 235 & 184 & 300 & 252 & & 138 & 199 & 255 & 222 & 240 & 211 & & 204 & 110 & 152 & 214 & 166 & 169 & \\
\hline & & \pm 42 & \pm 27 & \pm 63 & \pm 31 & \pm 39 & \pm 19 & & \pm 16 & \pm 39 & \pm 38 & \pm 29 & \pm 52 & \pm 17 & \multirow[b]{2}{*}{$\begin{array}{c}\mathrm{HT} \\
(0.261)\end{array}$} & \pm 45 & \pm 27 & \pm 37 & \pm 28 & \pm 29 & \pm 16 & \multirow[b]{2}{*}{$\begin{array}{c}\mathrm{HT} \\
(0.120)\end{array}$} \\
\hline & Winter & $\begin{array}{l}316 \\
\pm 34\end{array}$ & $\begin{array}{c}319 \\
\pm 6\end{array}$ & $\begin{array}{c}338 \\
\pm 5\end{array}$ & $\begin{array}{l}250 \\
\pm 18\end{array}$ & $\begin{array}{l}338 \\
\pm 76\end{array}$ & $\begin{array}{l}312 \\
\pm 17\end{array}$ & $\begin{array}{c}\mathrm{HT} \\
(0.071)\end{array}$ & $\begin{array}{l}200 \\
\pm 62\end{array}$ & $\begin{array}{l}240 \\
\pm 27\end{array}$ & $\begin{array}{l}239 \\
\pm 35\end{array}$ & $\begin{array}{l}100 \\
\pm 15\end{array}$ & $\begin{array}{l}265 \\
\pm 47\end{array}$ & $\begin{array}{l}209 \\
\pm 20\end{array}$ & & $\begin{array}{c}394 \\
\pm 103\end{array}$ & $\begin{array}{l}256 \\
\pm 58\end{array}$ & $\begin{array}{l}211 \\
\pm 36\end{array}$ & $\begin{array}{l}325 \\
\pm 43\end{array}$ & $\begin{array}{l}288 \\
\pm 50\end{array}$ & $\begin{array}{l}295 \\
\pm 29\end{array}$ & \\
\hline & Summer & 410 & 386 & 236 & 249 & 285 & 313 & \multirow{4}{*}{$\begin{array}{c}S * \mathrm{ST} \\
(0.079)\end{array}$} & 397 & 319 & 272 & 227 & 205 & 284 & \multirow{4}{*}{$\begin{array}{c}\mathrm{S} * \mathrm{HT} \\
(0.059)\end{array}$} & 397 & 404 & 413 & 344 & 258 & 363 & \multirow{4}{*}{$\begin{array}{c}\mathrm{S} * \mathrm{HT} \\
(0.447)\end{array}$} \\
\hline & & \pm 39 & \pm 48 & \pm 47 & \pm 41 & \pm 91 & \pm 27 & & \pm 96 & \pm 25 & \pm 37 & \pm 21 & \pm 9 & \pm 24 & & \pm 84 & \pm 51 & \pm 48 & \pm 27 & \pm 52 & \pm 25 & \\
\hline & Total & 284 & 272 & 266 & 208 & 294 & 265 & & 227 & 249 & 256 & 190 & 238 & 232 & & 299 & 229 & 240 & 269 & 218 & 251 & \\
\hline & & \pm 28 & \pm 24 & \pm 23 & \pm 19 & \pm 32 & \pm 12 & & $\begin{array}{r} \pm 35 \\
69\end{array}$ & \pm 23 & $\begin{array}{r} \pm 19 \\
6.7\end{array}$ & \pm 17 & \pm 21 & \pm 11 & & \pm 40 & \pm 31 & \pm 28 & \pm 20 & \pm 22 & \pm 13 & \\
\hline \multirow[t]{5}{*}{$\begin{array}{l}\text { MPV } \\
\text { (fl) }\end{array}$} & SWM & $\begin{array}{c}6.8 \\
\pm 0.2\end{array}$ & $\begin{array}{c}7.0 \\
\pm 0.2\end{array}$ & $\begin{array}{c}6.3 \\
\pm 0.3\end{array}$ & $\begin{array}{c}6.9 \\
\pm 0.2\end{array}$ & $\begin{array}{c}6.8 \\
\pm 0.2\end{array}$ & $\begin{array}{c}6.8 \\
\pm 0.1\end{array}$ & \multirow{2}{*}{$\begin{array}{c}\mathrm{S} \\
(0.023)\end{array}$} & $\begin{array}{c}6.9 \\
\pm 0.1\end{array}$ & $\begin{array}{c}6.8 \\
\pm 0.3\end{array}$ & $\begin{array}{c}6.7 \\
\pm 0.4\end{array}$ & $\begin{array}{c}6.8 \\
\pm 0.2\end{array}$ & $\begin{array}{c}6.6 \\
\pm 0.3\end{array}$ & $\begin{array}{c}6.7 \\
\pm 0.1\end{array}$ & \multirow{2}{*}{$\begin{array}{c}\mathrm{S} \\
(0.017)\end{array}$} & $\begin{array}{l}7.0 \\
\pm 0.2\end{array}$ & $\begin{array}{r}6.9 \\
\pm 0.3\end{array}$ & $\begin{array}{c}6.7 \\
\pm 0.1\end{array}$ & $\begin{array}{c}7.3 \\
\pm 0.3\end{array}$ & $\begin{array}{c}7.1 \\
\pm 0.2\end{array}$ & $\begin{array}{c}7.0 \\
\pm 0.1\end{array}$ & \multirow{2}{*}{$\begin{array}{c}\mathrm{S} \\
(0.019)\end{array}$} \\
\hline & NEM & $\begin{array}{c}7.1 \\
\pm 0.2\end{array}$ & $\begin{array}{c}6.5 \\
\pm 0.1\end{array}$ & $\begin{array}{c}6.9 \\
\pm 0.2\end{array}$ & $\begin{array}{c}6.7 \\
\pm 0.1\end{array}$ & $\begin{array}{c}7.1 \\
\pm 0.3\end{array}$ & $\begin{array}{r}6.9 \\
\pm 0.1\end{array}$ & & $\begin{array}{c}7.5 \\
\pm 0.2\end{array}$ & $\begin{array}{r}6.9 \\
\pm 0.2\end{array}$ & $\begin{array}{c}6.8 \\
\pm 0.4\end{array}$ & $\begin{array}{c}6.0 \\
\pm 0.3\end{array}$ & $\begin{array}{c}6.7 \\
\pm 0.4\end{array}$ & $\begin{array}{c}6.8 \\
\pm 0.2\end{array}$ & & $\begin{array}{c}6.7 \\
\pm 0.2\end{array}$ & $\begin{array}{c}7.0 \\
\pm 0.2\end{array}$ & $\begin{array}{c}6.8 \\
\pm 0.1\end{array}$ & $\begin{array}{c}6.7 \\
\pm 0.3\end{array}$ & $\begin{array}{c}6.6 \\
\pm 0.1\end{array}$ & $\begin{array}{c}6.7 \\
\pm 0.1\end{array}$ & \\
\hline & Winter & $\begin{array}{c}6.2 \\
\pm 0.1\end{array}$ & $\begin{array}{c}6.3 \\
\pm 0.2\end{array}$ & $\begin{array}{c}7.5 \\
\pm 0.2\end{array}$ & $\begin{array}{c}6.7 \\
\pm 0.2\end{array}$ & $\begin{array}{c}6.4 \\
\pm 0.2\end{array}$ & $\begin{array}{c}6.6 \\
\pm 0.1\end{array}$ & $\begin{array}{c}\mathrm{HT} \\
(0.675)\end{array}$ & $\begin{array}{c}6.5 \\
\pm 0.3\end{array}$ & $\begin{array}{c}7.0 \\
\pm 0.3\end{array}$ & $\begin{array}{c}-6.9 \\
\pm 0.2\end{array}$ & $\begin{array}{l}7.0 \\
\pm 0.1\end{array}$ & $\begin{array}{l}7.0 \\
\pm 0.2\end{array}$ & $\begin{array}{c}-6.9 \\
\pm 0.1\end{array}$ & $\begin{array}{c}\mathrm{HT} \\
(0.722)\end{array}$ & $\begin{array}{l}7.5 \\
\pm 0.1\end{array}$ & $\begin{array}{l}-6.6 \\
\pm 0.2\end{array}$ & $\begin{array}{r}6.1 \\
\pm 0.2\end{array}$ & $\begin{array}{c}7.0 \\
\pm 0.1\end{array}$ & $\begin{array}{l}7.0 \\
\pm 0.2\end{array}$ & $\begin{array}{l}7.1 \\
\pm 0.1\end{array}$ & $\begin{array}{c}\mathrm{HT} \\
(0.384)\end{array}$ \\
\hline & $\begin{array}{c}\text { Summ } \\
\text { er }\end{array}$ & $\begin{array}{c}7.0 \\
\pm 0.2\end{array}$ & $\begin{array}{r}6.5 \\
\pm 0.3\end{array}$ & $\begin{array}{r}6.0 \\
\pm 0.3\end{array}$ & $\begin{array}{c}6.7 \\
\pm 0.2\end{array}$ & $\begin{array}{c}6.0 \\
\pm 0.4\end{array}$ & $\begin{array}{c}6.4 \\
\pm 0.1\end{array}$ & \multirow{2}{*}{$\begin{array}{c}\mathrm{S} * \mathrm{HT} \\
(0.000)\end{array}$} & $\begin{array}{c}6.5 \\
\pm 0.1\end{array}$ & $\begin{array}{c}6.1 \\
\pm 0.2\end{array}$ & $\begin{array}{c}6.4 \\
\pm 0.1\end{array}$ & $\begin{array}{c}6.5 \\
\pm 0.1\end{array}$ & $\begin{array}{r}6.6 \\
\pm 0.3\end{array}$ & $\begin{array}{c}6.4 \\
\pm 0.1\end{array}$ & \multirow{2}{*}{$\begin{array}{l}\mathrm{S}^{*} \mathrm{HT} \\
(0.077)\end{array}$} & $\begin{array}{c}6.7 \\
\pm 0.2\end{array}$ & $\begin{array}{c}6.7 \\
\pm 0.2\end{array}$ & $\begin{array}{c}6.6 \\
\pm 0.1\end{array}$ & $\begin{array}{c}6.7 \\
\pm 0.2\end{array}$ & $\begin{array}{c}6.6 \\
\pm 0.2\end{array}$ & $\begin{array}{c}6.6 \\
\pm 0.1\end{array}$ & \multirow{2}{*}{$\begin{array}{l}\mathrm{S}^{*} \mathrm{HT} \\
(0.242)\end{array}$} \\
\hline & Total & $\begin{array}{c}6.8 \\
\pm 0.1\end{array}$ & $\begin{array}{c}6.6 \\
\pm 0.1\end{array}$ & $\begin{array}{c}-6.7 \\
\pm 0.2\end{array}$ & $\begin{array}{c} \pm .2 \\
0.1 \\
\pm 0.1\end{array}$ & $\begin{array}{c}-6.7 \\
\pm 0.2 \\
\pm 0.2\end{array}$ & $\begin{array}{c}-6.1 \\
\pm 0.1\end{array}$ & & $\begin{array}{c} \pm .1 \\
0.1 \\
\pm 0.1\end{array}$ & $\begin{array}{c} \pm . .7 \\
\pm 0.1\end{array}$ & $\begin{array}{r}-6.1 \\
\pm 0.1\end{array}$ & $\begin{array}{r} \pm .1 \\
\pm 0.1 \\
\pm 0.1\end{array}$ & $\begin{array}{c}6.7 \\
6.7 \\
+0.1\end{array}$ & $\begin{array}{c}6.1 \\
6.7 \\
+0.1\end{array}$ & & $\begin{array}{l}7.0 \\
+0.1\end{array}$ & $\begin{array}{c}-6.2 \\
6.8 \\
+0.1\end{array}$ & $\begin{array}{c}6.7 \\
+0.1\end{array}$ & $\begin{array}{c}6.2 \\
6.9 \\
+0.1\end{array}$ & $\begin{array}{c}-6.2 \\
6.8 \\
+0.1\end{array}$ & $\begin{array}{c}-3.1 \\
6.8 \\
+0.1\end{array}$ & \\
\hline
\end{tabular}

S. Season; HT - Housing type; S*HT - Season verses Housing interaction. Figures in parenthesis indicates significance 
Table.6 Zone wise details of Mean \pm SE of PDW and PCT in various housing types during South West monsoon (SWM), North East monsoon (NEM), Winter and Summer seasons

\begin{tabular}{|c|c|c|c|c|c|c|c|c|c|c|c|c|c|c|c|c|c|c|c|c|c|c|}
\hline \multirow[t]{4}{*}{ Name } & \multirow{4}{*}{ Seasons } & \multicolumn{21}{|c|}{ Agro Climatic Zones } \\
\hline & & \multirow{2}{*}{\multicolumn{7}{|c|}{$\begin{array}{c}\text { Southern } \\
\text { Housing Type }\end{array}$}} & \multirow{2}{*}{\multicolumn{7}{|c|}{$\begin{array}{l}\text { Cauvery Delta } \\
\text { Housing Type }\end{array}$}} & \multirow{2}{*}{\multicolumn{7}{|c|}{$\begin{array}{l}\text { High Rainfall } \\
\text { Housing Type }\end{array}$}} \\
\hline & & & & & & & & & & & & & & & & & & & & & & \\
\hline & & Thatched & Tiled & Asbestos & Metal & Open & Total & Anova & Thatched & Tiled & Asbestos & Metal & Open & Total & Anova & Thatched & Tiled & Asbestos & Metal & Open & Total & Anova \\
\hline \multirow{7}{*}{$\begin{array}{l}\text { PDW } \\
(\%)\end{array}$} & SWM & 15.1 & 15.2 & 15.3 & 15.3 & 15.8 & 15.3 & \multirow{2}{*}{$\begin{array}{c}\text { S } \\
(0.715)\end{array}$} & 15.3 & 15.0 & 15.3 & 15.3 & 15.0 & 15.2 & \multirow{2}{*}{$\begin{array}{c}\text { S } \\
(0.787)\end{array}$} & 15.7 & 15.4 & 15.3 & 15.8 & 15.5 & 15.5 & \multirow{2}{*}{$\begin{array}{c}\mathrm{S} \\
(0.840)\end{array}$} \\
\hline & & \pm 0.2 & \pm 0.2 & \pm 0.2 & \pm 0.2 & \pm 0.3 & \pm 0.1 & & \pm 0.2 & \pm 0.3 & \pm 0.3 & \pm 0.1 & \pm 0.3 & \pm 0.1 & & \pm 0.2 & \pm 0.2 & \pm 0.1 & \pm 0.3 & \pm 0.2 & \pm 0.1 & \\
\hline & NEM & $\begin{array}{l}14.1 \\
\pm 1.6\end{array}$ & $\begin{array}{l}14.9 \\
\pm 0.3\end{array}$ & $\begin{array}{l}15.3 \\
\pm 0.2\end{array}$ & $\begin{array}{l}15.3 \\
\pm 0.2\end{array}$ & $\begin{array}{l}15.6 \\
\pm 0.3\end{array}$ & $\begin{array}{l}15.0 \\
\pm 0.3\end{array}$ & \multirow{2}{*}{$\begin{array}{c}\mathrm{HT} \\
(0.290)\end{array}$} & $\begin{array}{l}15.9 \\
\pm 0.2\end{array}$ & $\begin{array}{l}15.0 \\
\pm 0.4\end{array}$ & $\begin{array}{l}15.2 \\
\pm 0.5\end{array}$ & $\begin{array}{l}15.3 \\
\pm 0.1\end{array}$ & $\begin{array}{l}15.4 \\
\pm 0.5\end{array}$ & $\begin{array}{l}15.4 \\
\pm 0.2\end{array}$ & \multirow{5}{*}{$\begin{array}{c}\mathrm{HT} \\
(0.843) \\
\mathrm{S}^{*} \mathrm{HT} \\
(0.452)\end{array}$} & $\begin{array}{l}15.2 \\
\pm 0.1\end{array}$ & $\begin{array}{l}15.7 \\
\pm 0.2\end{array}$ & $\begin{array}{l}15.8 \\
\pm 0.1\end{array}$ & $\begin{array}{l}15.7 \\
\pm 0.2\end{array}$ & $\begin{array}{l}15.5 \\
\pm 0.2\end{array}$ & $\begin{array}{l}15.6 \\
\pm 0.1\end{array}$ & \multirow{5}{*}{$\begin{array}{c}\mathrm{HT} \\
(0.164) \\
\mathrm{S}^{*} \mathrm{HT} \\
(0.009)\end{array}$} \\
\hline & Winter & 15.2 & 15.1 & 15.7 & 15.4 & 15.2 & 15.3 & & 14.4 & 15.5 & 15.3 & $\begin{array}{l} \pm 0.1 \\
15.9\end{array}$ & $\begin{array}{l} \pm 0.5 \\
15.6\end{array}$ & $\begin{array}{l} \pm 0.2 \\
15.3\end{array}$ & & $\begin{array}{l}0.1 \\
16.2\end{array}$ & $\begin{array}{l} \pm 0.2 \\
15.1\end{array}$ & $\begin{array}{l} \pm 0.1 \\
15.1\end{array}$ & $\begin{array}{l} \pm 0.2 \\
15.4\end{array}$ & $\begin{array}{l} \pm 0.2 \\
15.7\end{array}$ & $\begin{array}{l} \pm 0.1 \\
15.5\end{array}$ & \\
\hline & & \pm 0.2 & \pm 0.3 & \pm 0.2 & \pm 0.2 & \pm 0.2 & \pm 0.1 & \multirow{3}{*}{$\begin{array}{c}\mathrm{S}^{* \mathrm{HT}} \\
(0.918)\end{array}$} & \pm 1.4 & \pm 0.3 & \pm 0.2 & \pm 0.1 & \pm 0.2 & \pm 0.3 & & \pm 0.2 & \pm 0.1 & \pm 0.2 & \pm 0.2 & \pm 0.2 & \pm 0.1 & \\
\hline & Summer & $\begin{array}{l}15.1 \\
\pm 0.4\end{array}$ & $\begin{array}{l}15.1 \\
\pm 0.2\end{array}$ & $\begin{array}{l}15.1 \\
\pm 0.2\end{array}$ & $\begin{array}{l}15.4 \\
\pm 0.2\end{array}$ & $\begin{array}{l}15.3 \\
\pm 0.1\end{array}$ & $\begin{array}{l}15.2 \\
\pm 0.1\end{array}$ & & $\begin{array}{l}15.4 \\
\pm 0.1\end{array}$ & $\begin{array}{l}15.5 \\
\pm 0.1\end{array}$ & $\begin{array}{l}15.2 \\
\pm 0.1\end{array}$ & $\begin{array}{l}15.2 \\
\pm 0.1\end{array}$ & $\begin{array}{l}16.0 \\
\pm 0.3\end{array}$ & $\begin{array}{l}15.4 \\
\pm 0.1\end{array}$ & & $\begin{array}{l}15.6 \\
\pm 0.3\end{array}$ & $\begin{array}{l}15.6 \\
\pm 0.2\end{array}$ & $\begin{array}{l}15.3 \\
\pm 0.2\end{array}$ & $\begin{array}{l}15.3 \\
\pm 0.3\end{array}$ & $\begin{array}{l}15.6 \\
\pm 0.2\end{array}$ & $\begin{array}{l}15.5 \\
\pm 0.1\end{array}$ & \\
\hline & Total & $\begin{array}{l}14.9 \\
+0.4\end{array}$ & $\begin{array}{l}15.1 \\
+0.1\end{array}$ & 15.4 & 15.3 & 15.5 & 15.2 & & $\begin{array}{l}15.2 \\
+0.4\end{array}$ & 15.2 & 15.3 & 15.4 & $\begin{array}{l} \pm 0.3 \\
15.5 \\
+02\end{array}$ & $\begin{array}{l} \pm .1 \\
15.3 \\
+0.1\end{array}$ & & 15.7 & $\begin{array}{l}15.2 \\
15.4\end{array}$ & $\begin{array}{l} \pm .2 \\
15.3 \\
+0.1\end{array}$ & 15.5 & $\begin{array}{l}x_{0.2} \\
15.6 \\
+0.1\end{array}$ & $\begin{array}{l} \pm .1 \\
15.5 \\
+0.0\end{array}$ & \\
\hline \multirow[t]{5}{*}{$\begin{array}{l}\text { PCT } \\
(\%)\end{array}$} & SWM & $\begin{array}{c}0.07 \\
\pm 0.01\end{array}$ & $\begin{array}{c}0.09 \\
\pm 0.01\end{array}$ & $\begin{array}{c} \pm .15 \\
\pm 0.02\end{array}$ & $\begin{array}{c} \pm .10 \\
\pm 0.03 \\
\pm 0.03\end{array}$ & $\begin{array}{r}0.17 \\
\pm 0.033\end{array}$ & $\begin{array}{c} \pm 0.11 \\
\pm 0.01 \\
\pm 0.01\end{array}$ & \multirow{5}{*}{$\begin{array}{c}S \\
(0.000) \\
H T \\
(0.176) \\
S^{*} H T \\
(0.010)\end{array}$} & $\begin{array}{c}0.11 \\
\pm 0.01\end{array}$ & $\begin{array}{c} \pm 0.11 \\
0.11 \\
\pm 0.03\end{array}$ & $\begin{array}{c}0.16 \\
\pm 0.02\end{array}$ & $\begin{array}{l}0.13 \\
\pm 0.0\end{array}$ & $\begin{array}{c} \pm 0.15 \\
\pm 0.03\end{array}$ & $\begin{array}{c}0.13 \\
\pm 0.01\end{array}$ & \multirow{5}{*}{$\begin{array}{c}\mathrm{S} \\
(0.003) \\
\mathrm{HT} \\
(0.024) \\
\mathrm{S} * \mathrm{HT} \\
(0.005)\end{array}$} & $\begin{array}{c} \pm .13 \\
\pm 0.03 \\
\pm 0.03\end{array}$ & $\begin{array}{r}0.13 \\
\pm 0.01\end{array}$ & $\begin{array}{c} \pm .12 \\
\pm 0.02\end{array}$ & $\begin{array}{c}0.13 \\
\pm 0.01\end{array}$ & $\begin{array}{c} \pm 0.11 \\
0.11 \\
\pm 0.01\end{array}$ & $\begin{array}{c}0.12 \\
\pm 0.01\end{array}$ & \multirow{5}{*}{$\begin{array}{c}\mathrm{S} \\
(0.000) \\
\mathrm{HT} \\
(0.185) \\
\mathrm{S}^{*} \mathrm{HT} \\
(0.614)\end{array}$} \\
\hline & NEM & $\begin{array}{c}0.21 \\
\pm 0.02\end{array}$ & $\begin{array}{c}0.13 \\
\pm 0.01\end{array}$ & $\begin{array}{c}0.15 \\
\pm 0.05\end{array}$ & $\begin{array}{c}0.12 \\
\pm 0.02\end{array}$ & $\begin{array}{c}0.20 \\
\pm 0.02\end{array}$ & $\begin{array}{c}0.16 \\
\pm 0.01\end{array}$ & & $\begin{array}{c}0.10 \\
\pm 0.01\end{array}$ & $\begin{array}{c}0.13 \\
\pm 0.03\end{array}$ & $\begin{array}{c}0.19 \\
\pm 0.02\end{array}$ & $\begin{array}{l}2 \\
0.12 \\
\pm 0.0 \\
3\end{array}$ & $\begin{array}{c}0.15 \\
\pm 0.03\end{array}$ & $\begin{array}{c}0.14 \\
\pm 0.01\end{array}$ & & $\begin{array}{c}0.13 \\
\pm 0.02\end{array}$ & $\begin{array}{c}0.07 \\
\pm 0.02\end{array}$ & $\begin{array}{c}0.10 \\
\pm 0.02\end{array}$ & $\begin{array}{c}0.15 \\
\pm 0.02\end{array}$ & $\begin{array}{c}0.09 \\
\pm 0.02\end{array}$ & $\begin{array}{c}0.11 \\
\pm 0.01\end{array}$ & \\
\hline & Winter & $\begin{array}{l}0.19 \\
\pm 0.01\end{array}$ & $\begin{array}{c}0.18 \\
\pm 0.00\end{array}$ & $\begin{array}{c}0.19 \\
\pm 0.00\end{array}$ & $\begin{array}{c}0.17 \\
\pm 0.01\end{array}$ & $\begin{array}{c}0.15 \\
\pm 0.02\end{array}$ & $\begin{array}{c}0.18 \\
\pm 0.00\end{array}$ & & $\begin{array}{c}0.13 \\
\pm 0.03\end{array}$ & $\begin{array}{c}0.15 \\
\pm 0.01\end{array}$ & $\begin{array}{c}0.16 \\
\pm 0.02\end{array}$ & $\begin{array}{c}0.04 \\
\pm 0.0 \\
0\end{array}$ & $\begin{array}{c}0.14 \\
\pm 0.01\end{array}$ & $\begin{array}{c}0.13 \\
\pm 0.01\end{array}$ & & $\begin{array}{c}0.26 \\
\pm 0.08\end{array}$ & $\begin{array}{c}0.17 \\
\pm 0.03\end{array}$ & $\begin{array}{c}0.16 \\
\pm 0.02\end{array}$ & $\begin{array}{c}0.22 \\
\pm 0.03\end{array}$ & $\begin{array}{c}0.19 \\
\pm 0.03\end{array}$ & $\begin{array}{c}0.20 \\
\pm 0.02\end{array}$ & \\
\hline & Summer & $\begin{array}{l}0.27 \\
\pm 0.02\end{array}$ & $\begin{array}{c}0.25 \\
\pm 0.03\end{array}$ & $\begin{array}{l}0.15 \\
\pm 0.03\end{array}$ & $\begin{array}{l}0.16 \\
\pm 0.03\end{array}$ & $\begin{array}{l}0.17 \\
\pm 0.05\end{array}$ & $\begin{array}{c}0.20 \\
\pm 0.01\end{array}$ & & $\begin{array}{l}0.30 \\
\pm 0.06\end{array}$ & $\begin{array}{c}0.17 \\
\pm 0.00\end{array}$ & $\begin{array}{c}0.17 \\
\pm 0.02\end{array}$ & $\begin{array}{c}0.13 \\
\pm 0.0 \\
1\end{array}$ & $\begin{array}{l}0.16 \\
\pm 0.01\end{array}$ & $\begin{array}{c}0.19 \\
\pm 0.01\end{array}$ & & $\begin{array}{c}0.27 \\
\pm 0.06\end{array}$ & $\begin{array}{c}0.28 \\
\pm 0.03\end{array}$ & $\begin{array}{c}0.27 \\
\pm 0.03\end{array}$ & $\begin{array}{c}0.23 \\
\pm 0.01\end{array}$ & $\begin{array}{c}0.17 \\
\pm 0.03\end{array}$ & $\begin{array}{c}0.24 \\
\pm 0.01\end{array}$ & \\
\hline & Total & $\begin{array}{c}0.18 \\
\pm 0.01\end{array}$ & $\begin{array}{c}0.16 \\
\pm 0.01\end{array}$ & $\begin{array}{c}0.16 \\
\pm 0.01\end{array}$ & $\begin{array}{r}0.14 \\
\pm 0.01\end{array}$ & $\begin{array}{l}0.17 \\
\pm 0.01\end{array}$ & $\begin{array}{c}0.16 \\
\pm 0.00\end{array}$ & & $\begin{array}{l}0.16 \\
\pm 0.02\end{array}$ & $\begin{array}{l}0.14 \\
\pm 0.01\end{array}$ & $\begin{array}{c}0.17 \\
\pm 0.01\end{array}$ & $\begin{array}{c}0.11 \\
\pm 0.01\end{array}$ & $\begin{array}{c}0.15 \\
\pm 0.01\end{array}$ & $\begin{array}{c}0.15 \\
\pm 0.00\end{array}$ & & $\begin{array}{l}0.20 \\
\pm 0.03\end{array}$ & $\begin{array}{c}0.16 \\
\pm 0.02\end{array}$ & $\begin{array}{l}0.16 \\
\pm 0.01\end{array}$ & $\begin{array}{c}0.18 \\
\pm 0.01\end{array}$ & $\begin{array}{c}0.14 \\
\pm 0.01\end{array}$ & $\begin{array}{c}0.17 \\
\pm 0.00\end{array}$ & \\
\hline
\end{tabular}

S. Season; HT - Housing type; S*HT - Season verses Housing interaction. Figures in parenthesis indicates significance 
In the present study, it was evident that recorded total mean value of RDW were found between $(16.9 \pm 0.2$ to $17.7 \pm 0.2 \%)$. During South West monsoon season asbestos shed in Southern zone recorded higher RDW $(18.7 \pm 0.5 \%)$, whereas, tiled shed in Southern

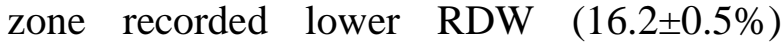
during North East monsoon. Observed values from this study were within the normal range only (Darren Wood and Gerado F. Quiroz Rocha, 2010). Similar finding was reported by Zeki and Kadir (2008).

\section{PLT Count}

The mean \pm S.E of PLT of animals under various housing types in Southern, Cauvery delta and High rainfall zone are presented in the Table 5. From the present study, it was evident that the PLT level between seasons was found to be less significant $(\mathrm{P}<0.05)$ in Cauvery delta zone, whereas it was found to be highly significant $(\mathrm{P}<0.01)$ in Southern and High rainfall zones. High rain fall zone recorded both higher PLT $\left(413 \pm 4810^{3} / \mu 1\right)$ and lower PLT $\left(152 \pm 3710^{3} / \mu 1\right)$ in asbestos shed during summer and North East monsoon seasons respectively. In general, the observed value of PLT in all the seasons was within normal range (Darren Wood and Gerado F. Quiroz Rocha, 2010).

\section{MPV}

The mean \pm S.E of MPV of animals under various housing types in Southern, Cauvery delta and High rainfall zone are presented in the Table 5. It was evident from the table 5 significant difference in MPV level was observed $(\mathrm{P}<0.05)$ between the seasons in all three zones. Thatched housing type in High rain fall zone and asbestos type in Southern zone recorded both higher MPV $7.5 \pm 0.1 \mathrm{fl}$ and $7.5 \pm 0.2 \mathrm{fl}$ respectively during Winter season and the lower value of MPV (6.0 \pm $0.4 \mathrm{fl})$ was recorded in open shed during Summer in Southern zone.

\section{PDW and PCT value}

The mean \pm S.E of PDW of animals under various housing types in Southern, Cauvery delta and High rainfall zone are presented in the Table 6. The observed PDW and PCT of animals recorded under various housing types in Southern, Cauvery delta and high rainfall zone are presented in the table 6. Higher PDW $(16.2 \pm 0.2 \%)$ was recorded in thatched shed during winter season in High Rainfall zone. The lower value of PDW $(14.1 \pm 1.6 \%)$ was recorded in thatched shed during North East monsoon season in Southern zone.

Tiled shed in high rain fall zone recorded higher PCT $(0.28 \pm 0.03 \%)$ during summer season. The lower value of PCT $(0.04 \pm 0.00 \%)$ was recorded in metal shed during winter season in Cauvery delta zone. The recorded values of PDW and PCT were found to be in the normal range (Darren Wood and Gerado F. Quiroz Rocha, 2010).

\section{References}

Banerjee D., and Ashutosh, 2010. Effect of thermal exposure on diurnal rhythms of physiological parameters and feed, water intake in Tharparkar and Karan Fries heifers. Biological Rhythm Research. 42(1): 39-51.

Coles, E.H., 1986. Veterinary clinical pathology. $4^{\text {th }}$ ed., W.B. Saunders Company, Philadelphia. Pp. 13-65.

Darren Wood and Gerado F. Quiroz Rocha, 2010. Normal Hematolgy of cattle. In: Douglas J Wess and K. Jane Wardrop (Ed.) Schalm's Veterinary Hematology, Wiley-Blackwell, USA, 829-835.

Grunwaldt, E.G., J.C. Guevara, O.R. Estevez, A. Vicente, H. Rousselle, N. Alcuten, D. Aguerregaray, and C.R. Stasi, 2005.Biochemical and haematological measurements in beef cattle in Mendoza plain rangelands (Argentina). Trop. Anim. Health and Prod. 37(6): 527-540. 
Perumal, P., S.Das, D.N. Mohanty, A.K. Barik, P.C. Mishra, and J.B. Pattnaik, 2010. Haematological studies in crossbred anoestrous cows. Indian $J$. Field Vet. 6(2): 7-10.

Randhawa, C. S., P. Heigo Pal, Randhawa S. S., and S. K. Uppal, 2009. Influence of age, season, lactation on haematology and iron biochemistry of crossbred cattle. Indian J. Anim. Sci. 79(10): 1007-1010.

Sastry,N.S.R., M.S. Yadav, and R.S. Yadav, 1988. Systems of keeping Murrah buffaloes in their home tracts. Proceedings of II World Buffaloe congress, New Delhi, 12-16 December,4: 336-345.

Sharma, M. C., J. Chinmay, and D. Gunjan, 2009. Soil, fodder and serum mineral (cattle) and haematobiochemical profile in some districts of Central Uttar Pradesh Indian J. Anim. Sci. 79(4): 411415.

Sinha, R. R. K., Triveni Dutt, Singh R. R, Bharat Bhushan, Mukesh Singh and Sanjay Kumar, 2009. Production and reproduction profile of cattle and buffaloes in Bareilly district of Uttar Pradesh. Indian J. Anim. Sci. 79 (8): 829-833.

Srikandakumar, A., and E.H. Johnson, 2004. Effect of Heat Stress on Milk Production, Rectal Temperature, Respiratory Rate and Blood Chemistry in Holstein, Jersey and Australian Milking Zebu Cows. Trop. Anim. Health Prod. 36(7): 685-692.

Weldy, J.R., R. E. McDowell, P. J. Van Soest, and J. Bond, 1964. Influence of heat stress on rumen acid levels and some blood constituents in cattle. J. Anim. Sci. 23: 147-153.

Whitaker, D.A., 2000. Use and interpretation of metabolic profiles. In: A.H. Andrews (ed.) The health of dairy cattle, Blackwell Science, Oxford, UK, 89-107

Zeki, Y. and Y. Kadir, 2008. Clinical and haematological findings in bovine immunodeficiency virus (BIV) infected cattle. Turk. J. Vet. Anim. Sci. 32(3): 207-214.

\section{How to cite this article:}

Paramasivam, A., P.S.L. Sesh, T. Sivakumar, P. Tensingh Gnanaraj and Suraj, P.T. 2019. Impact of Different Shelter Designs on Haematological Profile in Dairy Cattle of Tamil Nadu, India. Int.J.Curr.Microbiol.App.Sci. 8(02): 1068-1079. doi: https://doi.org/10.20546/ijcmas.2019.802.126 